\title{
A Perturbation Analysis of an Interaction between Long and Short Surface Waves
}

\author{
By S. L. Woodruff and A. F. Messiter
}

The interaction of finite-amplitude long gravity waves with a small-amplitude packet of short capillary waves is studied by a multiple-scale method based on the invariance of the perturbation expansion under certain translations. The result of the analysis is a set of equations coupling the complex amplitude of the packet of short waves with the long-wave velocity potential and surface elevation. The short wave is described by a Ginzburg-Landau equation with coefficients that depend on properties of the long wave. The long-wave potential and surface elevation satisfy the usual free-surface conditions augmented by forcing terms representing effects of the short waves. The derivation removes some of the restrictions imposed in earlier studies.

\section{Introduction}

While surface water waves have been studied since the days of Stokes [1] and before, there has been renewed interest lately, in part in the context of wakes and their sensing by radar (e.g., [2]). In particular, it is known that radar of relatively short wavelength can "see" water waves of much longer wavelength, but the mechanism that permits this is still not clear. It would also be useful to have analytical solutions describing interactions of long and short waves that would allow numerical analysts to account for the effects of short waves without having to resolve the smaller scales.

The earliest study of weakly nonlinear water waves was made by Stokes [1] for a train of waves at a single frequency. In a first approximation, he found that the effect of a small nonlinearity is to shift the frequency of the

Address for correspondence: Professor S. L. Woodruff, Division of Engineering, Brown University, Providence, RI 02912. 
waves by a small amount. Longuet-Higgins and Stewart [3] used a similar technique to investigate the interaction of trains of long and short waves, both linearized about the surface rest position.

More complex systems of waves were studied by Zakharov [4], who derived what is now known as the nonlinear Schrödinger equation to describe the evolution of a narrow-banded packet of two-dimensional surface waves. Benney and Roskes [5] showed that a three-dimensional packet of waves governed by the Navier-Stokes equations carries with it a largerscale (mean-flow) disturbance (see also [6]); this was shown specifically for the case of water waves by Davey and Stewartson [7]. Investigations of the interaction between long and short water waves are essentially extensions of this work.

Most of the more recent work in the interaction of long and short water waves may be categorized according to the family of perturbation techniques employed and where the surface conditions are applied. Perturbation techniques employed fall either into the category of multiple-variable methods or wave-action conservation principles. The question of where to apply the surface conditions is a particularly vexing one in the analysis of long and short waves: one would like to expand the surface conditions about the surface rest position, as is customary in perturbative analyses of water waves, but to do so imposes the severe restriction that the long-wave amplitude be small compared to the short-wave wavelength. This approach was employed in the work cited above, as well as by Benney [8], who used a multiplevariable method to derive amplitude equations describing the interaction of a linearized long gravity wave with a packet of weakly nonlinear short capillary waves.

The problem of how to study long waves with larger amplitude was first addressed by Phillips [9], who used wave-conservation laws to analyze the evolution of a linearized group of short waves over a prescribed long wave in a system of coordinates for which the long-wave surface is a coordinate surface. The short-wave surface conditions were linearized about the longwave surface position; the long-wave surface conditions were presumed satisfied exactly, so there was no restriction on the long-wave amplitude. Numerical results were given by Longuet-Higgins [10] for the case where the short waves are linearized gravity waves. Henyey et al. [11] used a Hamiltonian formulation to extend the analysis to more general long waves and linearized gravity-capillary short waves. Grimshaw [12] has also used wave-action conservation principles to examine the evolution of narrowbanded packets of short waves on long waves and currents.

Zhang [13] and Zhang and Melville [14] used the transformed coordinates of Phillips [9] in a multiple-variable analysis of the interaction of long and short gravity waves. In their work, the long-wave slope is small, but not so small that expansion of the surface conditions about the surface rest position is possible. Naciri and Mei [15] also performed a multiple-scale analysis of long and short gravity-wave interactions; they used a Lagrangian description and made the long wave a Gerstner wave of finite slope. The present work is concerned with the interaction of an arbitrary finite- 
amplitude long gravity wave with a weakly nonlinear packet of short capillary waves; an earlier version was given by Woodruff [16].

A new perturbation method $[16,17]$ for multiple-scale problems is used in this paper. The method is based on an invariance condition and consists of two main steps. First, a regular perturbation expansion (including secular terms) valid in some small region is made at an arbitrary point in the larger region in which a solution is desired. Then an invariance condition is applied to this small-scale expansion to give the solution validity on the larger scale. The method thus differs in many respects from conventional methods for multiple-scale problems. While more examples would have to be worked out in order to clarify any anticipated advantages, the authors believe that the method is systematic and relatively straightforward, and it is our hope that the method may be found to offer added convenience at various stages in the formulation and solution of a new problem. The purpose of this paper is to give what we believe is an important application of the method to water-wave interactions, illustrating the power and versatility of the method.

Thus, in the following section, the wave-interaction problem to be solved and the asymptotic limit processes to be used are described. The small-scale expansion is derived in Section 3 with the help of the computer-algebra system Mathematica ${ }^{\mathrm{TM}}$, using a generating-function approach especially suited to computer solution. The equations for the solution valid on the large scale are determined by the invariance-condition method in Section 4, and the equations for the long wave, including effects of the short waves, are given in Section 5. Comparisons with previous work are given in Section 6, along with numerical results for a simple example and a brief application to the capillary-blockage problem.

\section{Formulation}

We wish to study the two-dimensional nonlinear interaction of a singlefrequency, finite-amplitude, surface gravity wave with a small-amplitude packet of capillary waves as in Figure 1. That is, the long wave is influenced by gravity in the first approximation, has amplitude of the same order as its

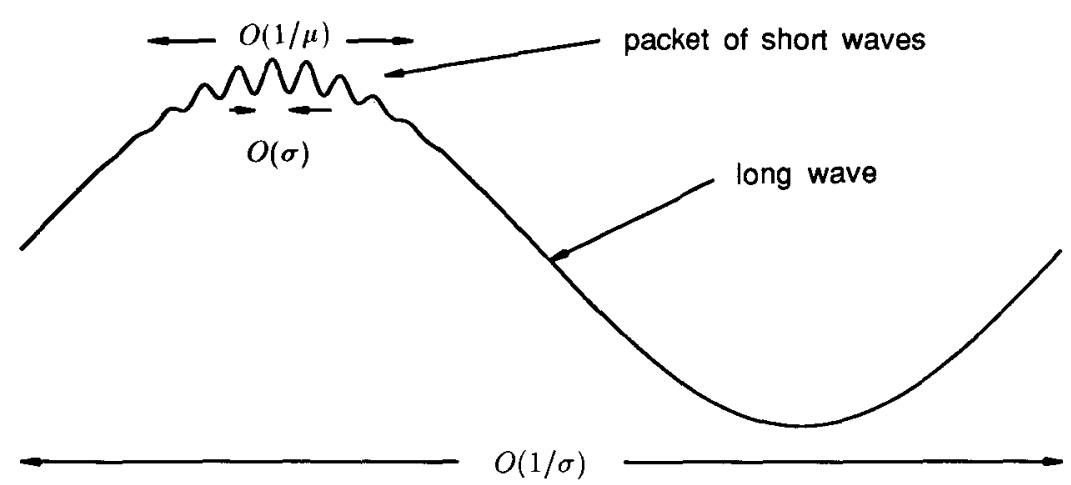

Figure 1. Sketch of configuration being analyzed. 
wavelength, and is described by the full nonlinear equations. The packet of short waves is influenced by surface tension in the first approximation and has an amplitude that is small in comparison with its typical wavelength. The short waves will be dealt with by the invariance-condition method. The result of the analysis will be a set of coupled nonlinear equations: the usual nonlinear gravity-wave equations for the long wave, augmented by terms representing the effect of the short waves on the long wave, and an amplitude equation for the short waves, obtained by the invariancecondition method, with variable coefficients depending on the long-wave flow quantities. The problem is greatly simplified by the assumption that the wavelength and period of the long wave are large in comparison with those of the short wave. If, however, short-scale variations develop on the long wave, as would occur near breaking, the present approximation would cease to ba valid. Thus, the formulation will be correct nearly up to the time when the wave breaks, and on those parts of the wave away from the broken crest.

The motion is assumed to be inviscid, incompressible, and irrotational. Coordinates $x$ and $y$ are defined, respectively, along and normal to the undisturbed free surface. The velocity potential $\phi$ satisfies Laplace's equation

$$
\phi_{x x}+\phi_{y y}=0
$$

for $-\infty<x<\infty$ and $-\infty<y<\eta$, where the free surface is defined by $y=\eta(x, t)$. Since disturbances decay at large depth,

$$
\phi_{x}^{2}+\phi_{y}^{2} \rightarrow 0 \quad \text { as } y \rightarrow-\infty .
$$

At the free surface, $y=\eta(x, t)$, the solution must satisfy the kinematic and dynamic conditions

$$
\begin{gathered}
\phi_{y}-\eta_{t}-\phi_{x} \eta_{x}=0 \\
\phi_{t}+\eta+\frac{1}{2}\left(\phi_{x}^{2}+\phi_{y}^{2}\right)-\eta_{x x}\left(1+\eta_{x}^{2}\right)^{-3 / 2}=0 .
\end{gathered}
$$

The equations have been made nondimensional with the length $(\rho g / T)^{-1 / 2}$ and the time $\left(\rho g^{3} / T\right)^{-1 / 4}$, where $T$ is the surface tension, $\rho$ is the fluid density, and $g$ is the gravitational acceleration. These length and time scales characterize a capillary-gravity wave, for which gravitational and surfacetension effects are of the same order, as is apparent from (2.4).

A small parameter $\sigma$ is defined as the ratio of the capillary-gravity length $(\rho g / T)^{-1 / 2}$ to a characteristic length of the long waves. In nondimensional terms, the long-wave length scale is then $1 / \sigma$. It follows from balancing orders of magnitude in the surface boundary conditions that, for the long wave, the nondimensional time scale is $1 / \sigma^{1 / 2}$, the potential $\phi$ is $O\left(1 / \sigma^{3 / 2}\right)$, and, since the amplitude is to be finite, the surface elevation $\eta$ is $O(1 / \sigma)$. 
The amplitude of the short-wave surface elevation, measured vertically from the long-wave surface, is characterized by a second small parameter $\epsilon$. We are interested in an interaction in which the long wave affects the short wave to first order. These long-wave terms, representing convection of the short wave by the long wave, may either dominate the gravity and surfacetension effects or balance one or both. If convective effects dominate, the short waves are nondispersive to first order, and a weakly nonlinear analysis would presumably lead to an amplitude equation similar to the Korteweg-de Vries equation. If the convection terms balance the linear restoring force (either gravity or surface tension), the short-wave problem is dispersive to first order, and a weakly nonlinear analysis leads to an amplitude equation of the nonlinear Schrödinger type. The latter case will be treated in the present paper.

A simple order-of-magnitude analysis reveal that balancing convective and gravitational effects requires that the magnitude of the long-wave slope vary like the square root of the ratio of short and long wavelengths, and so the long-wave slope must be small. Only by balancing the convective and surface-tension effects may the long-wave slope be of order one, as desired here. It is found that this balance leads to short-wave length and time scales that are $O(\sigma)$ and $O\left(\sigma^{3 / 2}\right)$, respectively, and the perturbation in the potential $\phi$ is $O\left(\epsilon / \sigma^{1 / 2}\right)$. This limiting case corresponds to a distinguished limit in the sense of Kevorkian and Cole [18] and is similar to that considered by Benney [8], but with larger wave amplitudes.

The short-wave problem is a singular-perturbation problem, since secular terms that grow with increasing time and/or distance will appear in higher approximations. Various methods exist for the derivation of uniformly valid solutions over ranges of time and distance that are large in comparison with the sort-wave scales. For example, a multiple-variable method [18] was used by Benney [8]. The present derivation is based on an invariance-condition method proposed by Woodruff $[16,17]$. This method provides an alternative way of extending the domain of validity of the solution to a multiple-scale problem. Secular terms are retained in this approach, and can be thought of as representing terms in Taylor expansions of the large-scale solution, whose form is constructed from certain invariance conditions.

The problem will be divided into a short-wave problem and a long-wave problem. Thus, the potential and surface elevation are written in the form

$$
\begin{gathered}
\phi=\frac{1}{\sigma^{3 / 2}} \bar{\phi}+\frac{\epsilon}{\sigma^{1 / 2}} \tilde{\phi} \\
\eta=\frac{1}{\sigma} \bar{\eta}+\epsilon \tilde{\eta},
\end{gathered}
$$

where the overbar and tilde denote the long-wave and short-wave quantities, respectively. The quantities $\bar{\phi}$ and $\bar{\eta}$ contain all parts of the solution with 
frequencies and wavenumbers asymptotically smaller than the short-wave fundamental, and the quantities $\tilde{\phi}$ and $\tilde{\eta}$ contain all the remaining terms.

The representations (2.5) and (2.6) can be substituted into (2.1) through (2.4) and each equation split into two equations. This is done by sorting terms according to whether they contain the short-wave length and time scales. As a result, the long-wave equations contain only terms with scales that are large in comparison with the short-wave fundamental, and the short-wave equations contain everything else. The short wave will be seen to be modulated by both intermediate and long length and time scales. The two sets of equations of course are coupled since products of short-wave quantities can contribute to the long-wave equation and products of long- and short-wave quantities can contribute to the short-wave equation.

To solve the short-wave problem, the invariance-condition method proceeds in two steps. First a "small-scale" expansion is constructed at an arbitrary point on the long wave. This is a regular perturbation expansion in terms of the smallest scales in the problem, the capillary-wave scales. Naturally, there will be some integration constants which cannot be determined since, in general, the region of validity of the small-scale expansion does not extend to the time or location at which initial or boundary conditions are imposed. These constants depend on the position on the long wave where the small-scale expansion is constructed. When this series is extended to higher orders, secular terms will appear that do not remain small in comparison with lower-order terms when the time or space variable becomes large. Whereas other methods eliminate these terms through the introduction of additional variables or by some transformation, the invariance-condition method uses the secular terms explicitly to construct the proper large-scale solution.

The second step of the method achieves this extension to larger scales by the following observation: if we have a small-scale expansion at a particular point on the long wave, and another expansion at a neighboring point within the domain of validity of the first expansion, then the two expansions must give the same value at any point where the regions of validity overlap. Requiring that this condition be satisfied determines the unknown constants in the small-scale expansion and completes the solution [17].

Formally, the extension is achieved by splitting each of the independent variables into two quantities,

$$
t=\frac{\bar{t}}{\tau}+\sigma^{3 / 2} \tilde{t}, \quad x=\frac{\bar{x}}{\lambda}+\sigma \tilde{x},
$$

where $\tau \ll 1$ and $\lambda \ll 1$. The barred quantities denote the point on the long wave where the small-scale expansion is constructed; the tilded quantities are the new variables for the small-scale expansion. The small parameters $\lambda$ and $\tau$ describe the extent of the large-scale region relative to the capillarygravity length and time scales used in the nondimensionalization. The observation concerning agreement of the two expansions is equivalent to the 
requirement that the small-scale expansion be invariant under the twoparameter translation group

$$
\begin{array}{cc}
\bar{x} \leftrightarrow \bar{x}+\lambda \sigma a, & \tilde{x} \leftrightarrow \tilde{x}-a \\
\bar{t} \leftrightarrow \bar{t}+\tau \sigma^{3 / 2} a^{\prime}, & \tilde{t} \leftrightarrow \tilde{t}-a^{\prime} .
\end{array}
$$

That is, suitably related displacements in the barred quantities and the tilded quantities should have cancelling effects and thus leave the solution unchanged. Clearly (2.7) is invariant under this transformation group. The condition that the small-scale expansion also be invariant under (2.8) may be enforced by substituting (2.8) into the expansion, differentiating with respect to $a$ and $a^{\prime}$, and setting the two results equal to zero. Use of a chain rule shows that this is equivalent to applying the operators

$$
\lambda \frac{\partial}{\partial \bar{x}}-\frac{1}{\sigma} \frac{\partial}{\partial \tilde{x}}, \quad \tau \frac{\partial}{\partial \dot{t}}-\frac{1}{\sigma^{3 / 2}} \frac{\partial}{\partial \tilde{t}}
$$

separately to the small-scale expansion and setting the results equal to zero. This procedure offers the most convenient way of using the method. The resulting equations will contain the small-scale variables $\tilde{x}$ and $\tilde{t}$; the equations must be satisfied for arbitrary values of $\tilde{x}$ and $\tilde{t}$.

At this stage, three sets of scales have been introduced. The nondimensional length and time scale for the short wave are $\sigma$ and $\sigma^{3 / 2}$, respectively, a solution for the short wave is sought that is valid on the large length scale $\lambda^{-1}$ and the larger time scale $\tau^{-1}$, and the characteristic length and time for the long wave are $\sigma^{-1}$ and $\sigma^{-1 / 2}$, respectively. If it is desired that the validity of the solutions $\tilde{\phi}$ and $\tilde{\eta}$ for the short wave be extended to the long-wave scales, the last two sets of scales should be the same, and we should choose

$$
\lambda=\sigma, \quad \tau=\sigma^{1 / 2}
$$

The spatial extent of the wave packet will be intermediate between the length scales $\sigma$ and $\sigma^{-1}$ of the short and long waves. This is typical of modulated-wave problems leading to an amplitude equation of the nonlinear Schrödinger type: if the ratio of large and small length and time scales is represented by a small parameter $\epsilon_{1}$, the spatial extent of the wavepacket will be $O\left(\epsilon_{1}^{-1 / 2}\right)$ relative to the short scale [18].

Equation (2.7) does not include a transformation for the $y$-coordinate since the short-wave disturbance merely decays in the $y$-direction on the short-wave length scale and so in a sense is confined to a boundary layer at the free surface. Away from the surface only a large-scale coordinate $\bar{y}$ is needed. A suitable small-scale variable $\tilde{y}$ is measured from the long-wave 
approximation for the free surface. Thus,

$$
\bar{y}=\sigma y, \quad y=\frac{1}{\sigma} \bar{\eta}+\sigma \tilde{y} .
$$

In each of the definitions (2.7), one or the other of the quantities is redundant and may be chosen arbitrarily. The barred quantities are parameters denoting the position of the short-scale expansion, and thus are considered to be constants in the small-scale expansion. Later, the above observation about invariance will allow unknown integration constants (which depend on these parameters) to be determined in such a way that the expansion can again be written in terms of the original variables $t$ and $x$. However, to short-cut some algebra at the end, we will simply set $\tilde{t}$ and $\tilde{x}$ equal to zero since they may be regarded as redundant, and use $\bar{t}$ and $\bar{x}$ as the new large-scale independent variables. In the same spirit, we will write, for example, $\bar{\eta}_{t}$ for the derivative of $\bar{\eta}$ with respect to the long-wave time coordinate and evaluated at $t=\bar{t} / \sigma^{1 / 2}$ and $x=\bar{x} / \sigma$. The dual usage of the barred quantities, as parameters for the small-scale solution and variables for the large-scale solution, simplifies the notation somewhat.

The procedure for applying the method have been described in simpler examples by Woodruff $[16,17]$. The tilded quantities are the appropriately scaled variables for the small-scale problem, and derivatives of $\phi$ and $\tilde{\eta}$ are taken with respect to these variables, with $\partial \tilde{\phi} / \partial x$ replaced by $\sigma^{-1} \partial \tilde{\phi} / \partial \tilde{x}$, etc. In addition, in the construction of the small-scale expansion, $\bar{\phi}$ and $\bar{\eta}$ will involve the tilded variables through (2.7) and (2.11). To account for the variation of the long-wave properties on the scale of the short wave, the slowly varying functions $\bar{\phi}$ and $\bar{\eta}$ are expanded in Taylor series about $t=\bar{t} / \sigma^{1 / 2}, x=\bar{x} / \sigma$, and, in the case of $\bar{\phi}, y=\bar{y} / \sigma$. The coefficients in these series are just the derivatives of $\bar{\phi}$ and $\bar{\eta}$ referred to in the preceding paragraph. In the construction of the problem for the long wave, one could express the long-wave quantities $\bar{\phi}$ and $\bar{\eta}$ in terms of $t$ and $x$, again with the substitutions (2.7) and (2.11), but this would be pointless since including the short-wave scales while solving the long-wave problem would just give the Taylor series expansions of the long-wave solutions. Consequently, the long-wave problem will be set on its own length and time scales, which will be denoted by the same symbols as the parameters $\bar{t}, \bar{x}$, and $\bar{y}$, as noted above. Derivatives of $\bar{\phi}$ and $\bar{\eta}$ are then taken with respect to the barred variables, with $\partial \bar{\phi} / \partial x$ replaced by $\sigma \partial \bar{\phi} / \partial \bar{x}$, etc. It follows from (2.1) that each of $\bar{\phi}$ and $\tilde{\phi}$ satisfies Laplace's equation, in the appropriate variables:

$$
\begin{aligned}
& \bar{\phi}_{\bar{x} \bar{x}}+\bar{\phi}_{\bar{y} \bar{y}}=0 \\
& \tilde{\phi}_{\tilde{x} \tilde{x}}+\tilde{\phi}_{\tilde{y} \tilde{y}}=0 .
\end{aligned}
$$

We require that $\bar{\phi}$ and $\tilde{\phi}$ individually satisfy (2.2). 
Making the substitutions in the surface boundary conditions (2.3) and (2.4) is a little more involved. Since the short wave has small amplitude, these boundary conditions may be expanded about the long-wave surface elevation $\bar{\eta}$. When an expansion is made on the short-wave scale, $\bar{\eta}$ and $\bar{\phi}$ may be expanded in Taylor series since they are characterized only by larger scales, and the coefficients of these series will be evaluated at the selected point on the long wave. Thus, the surface conditions for the small-scale problem are obtained by introducing Taylor expansions of the long-wave quantities about the values $t=\bar{t} / \sigma^{1 / 2}$ and $x=\bar{x} / \sigma$.

We insert the definition (2.11) for $\tilde{y}$ into (2.6) for the free surface, expanded on the short-wave length and time scales using (2.7). The result is the equation for the free surface in the small-scale problem (with the long-wave mean surface subtracted):

$$
\tilde{y}=\bar{\eta}_{i} \tilde{t}+\bar{\eta}_{\bar{x}} \tilde{x}+\sigma^{2}\left(\frac{1}{2} \bar{\eta}_{\bar{t}} \tilde{t}^{2}+\bar{\eta}_{\bar{x} \bar{t}} \tilde{x} \tilde{t}+\frac{1}{2} \bar{\eta}_{\bar{x} \bar{x}} \tilde{x}^{2}\right)+\cdots+\frac{\epsilon}{\sigma} \tilde{\eta}
$$

Thus, the surface boundary conditions for the small-scale problem should be evaluated at the plane

$$
\tilde{y}=\bar{\eta}_{t} \tilde{t}+\bar{\eta}_{\bar{x}} \tilde{x}
$$

and expanded in the higher-order terms in $\epsilon \tilde{\eta} / \sigma$ and in the Taylor series for $\bar{\eta}$. This plane (2.15) is the tangent plane to the long-wave surface. Note that we must have $\epsilon \leqslant \sigma$ in order to expand in $\tilde{\eta}$ and avoid a fully nonlinear free-surface problem. This condition is required because $\epsilon$ was defined as the height of the short wave relative to the capillary-gravity length scale, while the condition that allows expansion in the surface elevation of the short wave is that its amplitude be small compared to the wavelength, which is $O(\sigma)$.

Making all these substitutions and expanding leads to the proper surface conditions, the terms in these equations are divided into equations for the long wave and equations for the short wave on the basis of the length and time scales that characterize them. Terms contributing to the long-wave equations include terms containing solely the long-wave functions $\phi$ and $\bar{\eta}$ and those including various products of factors that contain the shortwave functions $\tilde{\phi}$ and $\tilde{\eta}$. The largest terms in the long-wave equations are given by

$$
\begin{gathered}
\bar{\eta}_{\bar{t}}-\bar{\phi}_{\bar{y}}+\bar{\eta}_{\bar{x}} \bar{\phi}_{\bar{x}}+\cdots=0 \\
\bar{\phi}_{\bar{t}}+\bar{\eta}+\frac{1}{2}\left(\bar{\phi}_{\bar{x}}^{2}+\bar{\phi}_{\bar{y}}^{2}\right)+\cdots=0 .
\end{gathered}
$$


The remaining terms, characterized by the short-wave scales (among others), contribute to the short-wave equations. The largest terms in the short-wave equations are given by

$$
\begin{gathered}
\tilde{\eta}_{\bar{t}}-\tilde{\phi}_{\bar{y}}+\bar{\eta}_{\bar{x}} \tilde{\phi}_{\bar{x}}+\bar{\phi}_{\bar{x}} \tilde{\eta}_{\bar{x}}+\cdots=0 \\
\tilde{\phi}_{\bar{t}}-\left(1+\bar{\eta}_{\bar{x}}^{2}\right)^{-3 / 2} \tilde{\eta}_{\bar{x} \bar{x}}+\bar{\phi}_{\bar{x}} \tilde{\phi}_{\bar{x}}+\bar{\phi}_{\bar{y}} \tilde{\phi}_{\tilde{y}}+\cdots=0 .
\end{gathered}
$$

The distinction is especially straightforward in this case, where the short wave is a wave packet and is thus described locally by an oscillatory exponential. Here all terms containing this exponential contribute to the short-wave equations; those without it contribute to the long-wave equations.

The long-wave equations may thus be singled out and solved at the boundary $\bar{y}=\bar{\eta}$ in conjunction with Laplace's equation. Similarly, the shortwave equations may be found from the rest of the terms and solved at the surface (2.15) in conjunction with Laplace's equation. This solution is effected by means of the invariance-condition method, as shown in the next section.

\section{Construction of the small-scale expansion}

In view of the large number of terms in the surface conditions, it is convenient to fix the order of magnitude of $\epsilon$ in terms of $\sigma$ from the start. As a compromise between the desire to construct a solution with as large a region of validity as possible and the work required to calculate higher-order terms, we choose $\epsilon$ such that the cubic nonlinearities become important on the length and time scales of the long wave. Since the long-wave length and time scales are $O\left(1 / \sigma^{2}\right)$ with respect to the short-wave scales, we choose $\epsilon$ such that the order of the cubic terms relative to the lowest-order terms in the small-scale parts of the surface conditions is $O\left(\sigma^{2}\right)$. This criterion is found to give

$$
\epsilon=\sigma^{2} .
$$

The surface conditions for the small-scale problem, as noted above, are obtained by introducing Taylor expansions of (2.3) and (2.4), with $\bar{\phi}$ expanded about $\tilde{y}=\bar{\eta}_{i} \tilde{t}+\bar{\eta}_{\bar{x}} \tilde{x}$ according to (2.14) and the barred variables expanded about the point $(\bar{t}, \bar{x})$ according to $(2.7)$ and, in the case of $\bar{\phi}$, about $\bar{y}=\bar{\eta}$ using (2.6) and (2.11). Since the solutions will contain oscillatory exponentials, it can be anticipated that each quadratic term in $\tilde{\eta}$ and $\tilde{\phi}$ will contain a slowly varying part that should be omitted. These parts of the quadratic terms will be indicated by the notation \langle\rangle , which then represents a kind of average, and are subtracted. The resulting conditions, with terms 
Long and Short Surface Waves

169

$O(\sigma)$ and $O\left(\sigma^{2}\right)$ in (2.18) and (2.19) now shown explicitly, are

$$
\begin{aligned}
& \tilde{\eta}_{\tilde{t}}-\tilde{\phi}_{\bar{y}}+\bar{\eta}_{\bar{x}} \tilde{\phi}_{\tilde{x}}+\bar{\phi}_{\bar{x}} \tilde{\eta}_{\bar{x}} \\
& =-\sigma\left\{\left(\tilde{\phi}_{\tilde{x} \tilde{x}} \tilde{\eta}-\left\langle\tilde{\phi}_{\tilde{x} \tilde{x}} \tilde{\eta}\right\rangle\right)+\bar{\eta}_{\tilde{x}}\left(\tilde{\phi}_{\tilde{x} \tilde{y}} \tilde{\eta}-\left\langle\tilde{\phi}_{\tilde{x} \tilde{y}} \tilde{\eta}\right\rangle\right)+\left(\tilde{\eta}_{\tilde{x}} \tilde{\phi}_{\tilde{x}}-\left\langle\tilde{\eta}_{\tilde{x}} \tilde{\phi}_{\tilde{x}}\right\rangle\right)\right\} \\
& -\sigma^{2}\left\{\left(\bar{\phi}_{\bar{x} \bar{x}}+\bar{\eta}_{\bar{x}} \bar{\phi}_{\bar{x} \bar{y}}\right) \tilde{\eta}+\left(\bar{\eta}_{\bar{x} \bar{t}} \tilde{\phi}_{\tilde{x}}+\bar{\phi}_{\bar{x} \bar{t}} \tilde{\eta}_{\tilde{x}}\right) \tilde{t}+\left(\bar{\eta}_{\bar{x} \bar{x}} \tilde{\phi}_{\bar{x}}+\bar{\phi}_{\bar{x} \bar{x}} \tilde{\eta}_{\bar{x}}\right) \tilde{x}\right. \\
& +\tilde{\eta}_{\bar{x}} \bar{\phi}_{\bar{x} \bar{y}}\left(\bar{\eta}_{\bar{t}} \tilde{t}+\bar{\eta}_{\bar{x}} \tilde{x}\right)-\frac{1}{2}\left(\tilde{\phi}_{\tilde{y} \tilde{y} \tilde{y}}+\bar{\eta}_{\bar{x}} \tilde{\phi}_{\tilde{x} \tilde{x} \tilde{x}}\right) \tilde{\eta}^{2}+\tilde{\eta} \tilde{\eta}_{\bar{x}} \tilde{\phi}_{\tilde{x} \tilde{y}} \\
& \left.+\left(\tilde{\phi}_{\bar{x} \bar{x}}+\bar{\eta}_{\bar{x}} \tilde{\phi}_{\tilde{x} \bar{y}}\right)\left(\frac{1}{2} \bar{n}_{\bar{i} \bar{t}} \tilde{t}^{2}+\eta_{\bar{x} \bar{i}} \tilde{x} \tilde{t}+\frac{1}{2} \bar{\eta}_{\bar{x} \tilde{x}} \tilde{x}^{2}\right)\right\}
\end{aligned}
$$

$$
\begin{aligned}
& \tilde{\phi}_{\tilde{t}}-\kappa^{3} \tilde{\eta}_{\tilde{x} \tilde{x}}+\bar{\phi}_{\tilde{x}} \tilde{\phi}_{\tilde{x}}+\bar{\phi}_{\bar{y}} \tilde{\phi}_{\tilde{y}} \\
& =-\sigma\left\{\left(\tilde{\phi}_{i \bar{y}} \tilde{\eta}-\left\langle\tilde{\phi}_{i \tilde{y}} \tilde{\eta}\right\rangle\right)+\bar{\phi}_{\bar{x}}\left(\tilde{\phi}_{\tilde{x} \tilde{y}} \tilde{\eta}-\left\langle\tilde{\phi}_{\tilde{x} \tilde{y}} \tilde{\eta}\right\rangle\right)+\bar{\phi}_{\bar{y}}\left(\tilde{\phi}_{\bar{y} \tilde{y}} \tilde{\eta}-\left\langle\tilde{\phi}_{\bar{y} \tilde{y}} \tilde{\eta}\right\rangle\right)\right. \\
& \left.+\frac{1}{2}\left(\tilde{\phi}_{\tilde{x}}^{2}-\left\langle\tilde{\phi}_{\tilde{x}}^{2}\right\rangle\right)+\frac{1}{2}\left(\tilde{\phi}_{\bar{y}}^{2}-\left\langle\tilde{\phi}_{\tilde{y}}^{2}\right\rangle\right)+3 \kappa^{5} \bar{\eta}_{\bar{x}}\left(\tilde{\eta}_{\tilde{x}} \tilde{\eta}_{\tilde{x} \tilde{x}}-\left\langle\tilde{\eta}_{\tilde{x}} \eta_{\bar{x} \tilde{x}}\right\rangle\right)\right\} \\
& -\sigma^{2}\left\{\left(1+\bar{\phi}_{\bar{i} \bar{y}}+\bar{\phi}_{\bar{x}} \bar{\phi}_{\bar{x} \bar{y}}+\bar{\phi}_{\bar{y}} \bar{\phi}_{\bar{y} \bar{y}}\right) \tilde{\eta}+3 \kappa^{5} \bar{\eta}_{\bar{x}} \bar{\eta}_{\bar{x} \bar{x}} \tilde{\eta}_{\bar{x}}\right. \\
& +\left(\bar{\phi}_{\bar{x} \bar{t}} \tilde{\phi}_{\bar{x}}+\bar{\phi}_{\bar{y} \bar{i}} \tilde{\phi}_{\tilde{y}}+3 \kappa^{5} \bar{\eta}_{\bar{x}} \bar{\eta}_{\bar{x} \bar{t}} \tilde{\eta}_{\tilde{x} \tilde{x}}\right) \tilde{t} \\
& +\left(\bar{\phi}_{\bar{x} \bar{x}} \tilde{\phi}_{\tilde{x}}+\bar{\phi}_{\bar{x} \bar{y}} \tilde{\phi}_{\bar{y}}+3 \kappa^{5} \bar{\eta}_{\bar{x}} \bar{\eta}_{\bar{x} \bar{x}} \tilde{\eta}_{\tilde{x} \tilde{x}}\right) \tilde{x} \\
& +\left(\tilde{\phi}_{\tilde{x}} \tilde{\phi}_{\tilde{x} \tilde{y}}+\tilde{\phi}_{\tilde{y}} \tilde{\phi}_{\tilde{y} \tilde{y}}\right) \tilde{\eta}+\left(\tilde{\phi}_{\tilde{x}} \bar{\phi}_{\bar{x} \bar{y}}+\tilde{\phi}_{\tilde{y}} \bar{\phi}_{\bar{y} \tilde{y}}\right)\left(\bar{\eta}_{\bar{t}} \tilde{t}+\bar{\eta}_{\bar{x}} \tilde{x}\right) \\
& +\left(\tilde{\phi}_{i \tilde{y}}+\bar{\phi}_{\bar{x}} \tilde{\phi}_{\tilde{x} \tilde{y}}+\bar{\phi}_{\tilde{y}} \tilde{\phi}_{\dot{y} \tilde{y}}\right)\left(\frac{1}{2} \bar{\eta}_{\bar{t} t} \tilde{t}^{2}+\bar{\eta}_{\bar{x} \bar{i}} \tilde{x} \tilde{t}+\frac{1}{2} \bar{\eta}_{\bar{x} \bar{x}} \tilde{x}^{2}\right) \\
& +\frac{1}{2} \tilde{\eta}^{2}\left(\tilde{\phi}_{i \tilde{y} \tilde{y}}+\bar{\phi}_{\bar{x}} \tilde{\phi}_{\tilde{x} \tilde{y} \tilde{y}}+\bar{\phi}_{\bar{y}} \tilde{\phi}_{\tilde{y} \tilde{y} \tilde{y}}\right) \\
& \left.+\frac{3}{2} \kappa^{5}\left(1-5 \kappa^{2} \bar{\eta}_{\bar{x}}^{2}\right) \tilde{\eta}_{\tilde{x}}^{2} \tilde{\eta}_{\tilde{x} \tilde{x}}\right\}
\end{aligned}
$$


evaluated at the plane (2.15). We have defined $\kappa=\left(1+\bar{\eta}_{\bar{x}}^{2}\right)^{-1 / 2}$ for convenience; $\kappa$ is the cosine of the angle between the local long-wave surface and the horizontal.

The homogeneous first-order problem for the short waves may be solved in a manner similar to that for the standard capillary-wave solution, in spite of the need for imposing the surface conditions at the plane (2.15). The Fourier-integral solution may be written

$$
\begin{gathered}
\tilde{\eta}^{(1)}=\int_{-\infty}^{\infty} A e^{i\{\bar{k} \tilde{x}-\bar{\omega}(\bar{k}) \tilde{t}\}} d \bar{k}+\text { c.c. } \\
\tilde{\phi}^{(1)}=\int_{-\infty}^{\infty} C e^{i\left\{\bar{k}^{\prime} \bar{x}-\bar{\omega}\left(\bar{k}^{\prime}\right) \bar{t}^{\prime}\right.} e^{\bar{k}^{\prime} \tilde{y}} d \bar{k}+\text { c.c. }
\end{gathered}
$$

where c.c. denotes the complex conjugate; $\bar{k}$ and $\bar{\omega}$ are the wavenumber and frequency of the surface elevation; and $\bar{k}^{\prime}$ and $\bar{\omega}^{\prime}$ are the (different) wavenumber and frequency of the velocity potential (reflecting the fact that, for example, the potential must decay in a direction normal to the surface (2.15) rather than in a downward direction, as in the usual surface-wave problem). The potential (3.5) satisfies Laplace's equation in the short-scale tilded variables, and $A$ and $C$ are functions of the large-scale barred parameters.

The primed wavenumber and frequency are determined such that the argument of the exponential in $\tilde{\phi}^{(1)}$ is identical to the argument of the exponential in $\tilde{\eta}^{(1)}$ when $\tilde{\phi}^{(1)}$ is evaluated at the surface (2.15):

$$
\bar{k}^{\prime}=\frac{\bar{k}}{b}, \quad \bar{\omega}^{\prime}=\bar{\omega}-i \frac{\bar{k}}{b} \bar{\eta}_{t}
$$

where $b$ is defined by $b=1-i \bar{\eta}_{\bar{x}}$. The exponentials now cancel in the surface conditions. The solution may be completed in the customary manner, yielding the dispersion relation and a relation between $A$ and $C$ :

$$
\hat{\omega}=\kappa^{3 / 2} \bar{k}^{3 / 2}+\frac{1}{2} i \sigma^{2} \frac{\bar{k}}{b} F(\bar{x}, \bar{t}), \quad \bar{k} C=-i \hat{\omega} A
$$

where $\hat{\omega}$, the intrinsic frequency observed in coordinates fixed with respect to the long wave, is

$$
\hat{\omega}=\bar{\omega}-\bar{k} \bar{\phi}_{\bar{x}}
$$

The second term in the expression (3.7) for $\hat{\omega}$ arises because the long-wave kinematic surface condition (2.16) has been used to eliminate $\bar{\eta}_{t}$ and thus simplify the dispersion relation; the function $F(\bar{x}, \bar{t})$ is $O(1)$ and $-\sigma^{2} F(\bar{x}$, $\bar{t}$ ) represents the lowest-order term omitted from (2.16) (shown explicitly in 
(5.2)). Because we will keep terms only up to $O\left(\sigma^{2}\right)$, this additional contribution to (3.7) is only important when the dispersion relation is applied to the first term in the expansion. For consistency, we account for this effect by including the equivalent secular term in an expansion of the exponential in (3.4), namely

$$
\frac{1}{2} \sigma^{2} \frac{\bar{k}}{b} F(\bar{x}, \bar{t}) A \tilde{t} e^{i(\bar{k} \tilde{x}-\bar{\omega}(\bar{k}) \hat{t}\}}+\text { c.c. }
$$

and then consider $\hat{\omega}$ to be given by (3.7) without the second term on the right side, so that the dispersion relation becomes

$$
\hat{\omega}=\bar{\omega}-\bar{k} \bar{\phi}_{\bar{x}}=\kappa^{3 / 2} \bar{k}^{3 / 2} .
$$

That is, we incorporate this small correction to the frequency into the small-scale expansion by expanding the exponential in the small parameter $\sigma^{2}$, yielding an additional secular term, similar to other secular terms, to be calculated later, that will also affect the frequency.

Unless some further simplification is made, the higher-order nonlinear terms to be calculated will involve convolutions of the amplitude functions $A$ and $C$. As is common in weakly nonlinear analyses, we adopt the assumption that the initial condition for the short waves contains only waves with wavenumbers close to some particular value. This wave-packet assumption serves to break up the convolutions and leads to differential equations for the short-wave amplitudes, instead of integro-differential equations. Of course, it is possible for the wave packet to evolve into a solution containing wavenumbers over a larger region of wavenumber space, at which point the solution would no longer be valid.

Having made this final assumption, we summarize the assumptions made about the waves in this paper, assumptions characterized by the small parameters $\sigma, \epsilon$, and $\mu$ :

(1) the long-wave wavelength is $O(1 / \sigma)$ relative to the gravity-capillary length scale;

(2) the short-wave wavelength is $O(\sigma)$ relative to the gravity-capillary length scale;

(3) the short-wave surface elevation amplitude is $O(\epsilon)$;

(4) the band-width spectral distribution for the short wave is $O(\mu)$.

To construct the wave-packet expansion from the integrals (3.4) and (3.5), we make the substitutions

$$
\bar{k}=k+\mu k^{\prime}, \quad \bar{\omega}=\omega+\mu \omega_{k} k^{\prime}+\frac{1}{2} \mu^{2} \omega_{k k} k^{\prime 2}+\cdots,
$$

where $k$ denotes the position of the band of wavenumbers in wavenumber space, $\mu$ is a small parameter characterizing its width (the bandwidth), and we integrate over $k^{\prime}$. The frequency $\omega$ and its derivatives $\omega_{k}$ and $\omega_{k k}$ are 
related to the wavenumber by the dispersion relation and are evaluated at $k$, as are (3.9) and (3.10). The local frequency and wavenumber are also functions of the barred parameters since the dispersion relation depends explicitly on $\bar{x}$ and $\bar{t}$. This dependence of $k$ and $\omega$ must be taken into account when applying the invariance conditions. The expansion of (3.4) in $\mu$ is then

$$
\tilde{\eta}^{(1)}=A_{0} E+\mu i\left(\tilde{x}-\omega_{k} \tilde{t}\right) A_{1} E-\frac{1}{2} \mu^{2}\left\{\left(\tilde{x}-\omega_{k} \tilde{t}\right)^{2}+i \omega_{k k} \tilde{t}\right\} A_{2} E+\cdots+\text { c.c. }
$$

with a similar expansion for $\tilde{\phi}_{1}$. Here

$$
E=e^{i(k \tilde{x}-\omega \bar{t})}, \quad A_{j}=\mu \int k^{\prime j} A\left(k+\mu k^{\prime}\right) d k^{\prime} .
$$

Note that the $A_{j}$ are of order one; the function $A(k)$ must be $O(1 / \mu)$ in order that a finite amount of energy be contained in the bandwidth $\mu$. We retain the first three terms in the wave-packet expansion, and assume that $\mu=\sigma$, so that the smallest term retained in the wave-packet expansion is of the same order as the smallest terms retained elsewhere. The retention of more terms in the wave-packet expansion would give a solution valid for disturbances with larger bandwidth and contribute higher-order derivatives to the resulting amplitude equations.

We now expand $\tilde{\eta}$ and $\tilde{\phi}$ in the form

$$
\begin{aligned}
& \tilde{\eta}=\tilde{\eta}^{(1)}+\sigma \tilde{\eta}^{(2)}+\sigma^{2} \tilde{\eta}^{(3)}+O\left(\sigma^{3}\right) \\
& \tilde{\phi}=\tilde{\phi}^{(1)}+\sigma \tilde{\phi}^{(2)}+\sigma^{2} \tilde{\phi}^{(3)}+O\left(\sigma^{3}\right)
\end{aligned}
$$

To keep the notation from getting even more complex, we allow $\tilde{\eta}^{(1)}$ to contain the $O(\mu)$ and $O\left(\mu^{2}\right)$ terms of the wave-packet expansion ( $\mu$ is the wave-packet bandwidth as defined in (3.11)), but $\tilde{\eta}^{(2)}$ and $\tilde{\eta}^{(3)}$ will contain only the $O(\sigma)$ and $O\left(\sigma^{2}\right)$ terms, respectively. The same applies to terms in $\tilde{\phi}$. There are, of course, terms of order, $\mu \sigma$, etc., but these will have no effect on the result to the present order of approximation. All terms in $\tilde{\phi}$ are to satisfy Laplace's equation; equations relating the $O(\sigma)$ and $O\left(\sigma^{2}\right)$ terms at the surface (2.15) are found by substituting the expansions of $\tilde{\eta}$ and $\tilde{\phi}$ into (3.2) and (3.3). The conditions for the higher-order corrections may be written as

$$
\begin{gathered}
\tilde{\eta}_{t}^{(j)}-\tilde{\phi}_{\bar{y}}^{(j)}+\bar{\eta}_{\bar{x}} \tilde{\phi}_{\bar{x}}^{(j)}+\bar{\phi}_{\bar{x}} \tilde{\eta}_{\bar{x}}^{(j)}=P^{(j)} \\
\tilde{\phi}_{i}^{(j)}-\kappa^{3} \tilde{\eta}_{\bar{x} \bar{x}}^{(j)}+\bar{\phi}_{\bar{x}} \tilde{\phi}_{\bar{x}}^{(j)}+\bar{\phi}_{\bar{y}} \tilde{\phi}_{\bar{y}}^{(j)}=Q^{(j)}
\end{gathered}
$$


for $j=2,3$. The right-hand sides, given in the Appendix, contain terms proportional to $E, E^{2}, E^{3}$ and their complex conjugates; the coefficients of $E$ and $E^{-1}$ are polynomials in $\tilde{x}$ and $\tilde{t}$.

The series of problems with surface conditions given by (3.16) and (3.17) is solved by means of a generating function, which we now introduce and define. The problem with surface conditions

$$
\begin{gathered}
\tilde{\eta}_{\bar{t}}-\tilde{\phi}_{\bar{y}}+\bar{\eta}_{\bar{x}} \tilde{\phi}_{\bar{x}}+\bar{\phi}_{\bar{x}} \tilde{\eta}_{\bar{x}}=p e^{i n(k \tilde{x}-\omega \tilde{t})} e^{n(\alpha \tilde{x}-\beta \tilde{t})} \\
\tilde{\phi}_{\bar{t}}-\kappa^{3} \tilde{\eta}_{\bar{x} \bar{x}}+\bar{\phi}_{\bar{x}} \tilde{\phi}_{\tilde{x}}+\bar{\phi}_{\bar{y}} \tilde{\phi}_{\tilde{y}}=q e^{i n(k \bar{x}-\omega \bar{t})} e^{n(\alpha \bar{x}-\beta \tilde{t})}
\end{gathered}
$$

for arbitrary $\alpha$ and $\beta$ and for $n=0,1,2$, when expanded in Taylor series about $\alpha=\beta=0$ yields all possible secular right-hand sides of the form $\tilde{x}^{a} \tilde{t}^{b} e^{i n(k \tilde{x}-\omega \hat{t})}$. The argument of the second exponential in (3.18) and in (3.19) is real. If (3.18) and (3.19) are solved in conjunction with Laplace's equation for $\tilde{\phi}$, the resulting $\tilde{\eta}$ and $\tilde{\phi}$ are generating functions for all the secular solutions we shall need for different $a$ and $b$. The solution for $\tilde{\eta}$ is

$$
\tilde{\eta}=\frac{\left\{p\left(-i \hat{\omega}+\bar{\phi}_{\bar{x}} \alpha-\beta\right)+q(k-i \alpha)\right\} e^{i n(k \tilde{x}-\omega \tilde{t})} e^{n(\alpha \bar{x}-\beta \tilde{t})}}{n\left(-i \hat{\omega}+\alpha \bar{\phi}_{\bar{x}}-\beta\right)^{2}+n^{2} \kappa^{3}(k-i \alpha)^{3}}
$$

and a corresponding solution is found for $\tilde{\phi}$.

If the problem (3.18), (3.19) is expanded in $\alpha$ and $\beta$, the individual terms give the right-hand sides for the problem whose solutions we seek. Consequently, to find the required solutions, we simply take the corresponding terms in the expansion of (3.20) in $\alpha$ and $\beta$. A list of the solutions needed is given in the Appendix. It has been found convenient to change temporarily to the variables $\tilde{t}$ and $\xi=\tilde{x}-\omega_{k} \tilde{t}$ because these variables make is simpler to use the secular solutions of the homogeneous equations (which are in fact the $\mu$ and $\mu^{2}$ terms in the wavepacket expansion (3.12)) to give a small-scale expansion containing only secular terms linear in $\tilde{t}$, terms proportional to the terms in the wavepacket expansion (which may be absorbed into those terms), and some additional terms that do not affect the invariance conditions. This is done in order that the equations resulting from the application of the invariance conditions may be more easily transformed into familiar forms.

To solve (3.18) and (3.19), the generating-function solution for each type of term on the right side is taken from the Appendix, multiplied by the coefficient of that term, and added to the solution. Following this procedure, we find

$$
\begin{gathered}
\tilde{\eta}^{(2)}=k \kappa^{2}\left(-\frac{1}{2}+i \bar{\eta}_{\bar{x}}\right) A_{0}^{2} E^{2}+\text { c.c. } \\
\tilde{\eta}^{(3)}=f(\bar{x}, \bar{t}) \tilde{t} A_{0} E+g(\bar{x}, \bar{t}) \tilde{t} A_{0}^{2} A_{0}^{*} E+\cdots,
\end{gathered}
$$


where $A_{0}^{*}$ is the complex conjugate of $A_{0}$, and

$$
\begin{aligned}
f(\bar{x}, \bar{t})= & -\frac{i k}{2 \hat{\omega}}+\left\{\frac{15 \kappa^{2} \hat{\omega}}{8 k} \bar{\eta}_{\bar{x}}-i \frac{\kappa^{2}}{8}\left(1+7 i \bar{\eta}_{\bar{x}}\right) \bar{\phi}_{\bar{x}}-i \frac{\kappa^{2} k}{4 \hat{\omega}}\left(1+i \bar{\eta}_{\bar{x}}\right) \bar{\phi}_{\bar{x}}^{2}\right\} \bar{\eta}_{\bar{x} \bar{x}} \\
& -i\left\{\frac{\kappa^{2}}{8}\left(1+7 i \bar{\eta}_{\bar{x}}\right)+\frac{\kappa^{2} k}{2 \hat{\omega}}\left(1+i \bar{\eta}_{\bar{x}}\right) \bar{\phi}_{\bar{x}}\right\} \bar{\eta}_{\bar{x} \bar{t}} \\
& -i \frac{\left(1+i \bar{\eta}_{\bar{x}}\right) \kappa^{2} k}{4 \hat{\omega}} \bar{\eta}_{\bar{i} \bar{t}}+\left\{-\frac{1}{4} \kappa^{2}\left(1+i \bar{\eta}_{\bar{x}}\right)+\frac{k}{4 \hat{\omega}}\left(1-\kappa^{2}-i \kappa^{2} \bar{\eta}_{\bar{x}}\right) \bar{\phi}_{\bar{x}}\right. \\
& \left.+\frac{i k}{4 \hat{\omega}}\left(2-\kappa^{2}-i \kappa^{2} \bar{\eta}_{\bar{x}}\right) \bar{\phi}_{\bar{y}}\right\} \bar{\phi}_{\bar{x} \bar{x}} \\
& +\left\{-\frac{1}{8} i\left(1-i \bar{\eta}_{\bar{x}}\right)+\frac{1}{4} i \kappa^{2}\left(1+i \bar{\eta}_{\bar{x}}\right)-\frac{i k}{4 \hat{\omega}}\left(2-\kappa^{2}-i \kappa^{2} \bar{\eta}_{\bar{x}}\right) \bar{\phi}_{\bar{x}}\right. \\
& \left.+\frac{k \hat{\omega}}{4 \hat{\omega}}\left(1-\kappa^{2}-i \kappa^{2} \bar{\eta}_{\bar{x}}\right) \bar{\phi}_{\bar{y}}\right\} \bar{\phi}_{\bar{x} \bar{y}} \\
& g(\bar{x}, \bar{t})=\frac{1}{4} i \kappa^{4} k^{2} \hat{\omega}+\kappa^{2} k_{\bar{x}}^{2} \hat{\omega} \frac{\left(1+i \bar{\phi}_{\bar{x} \bar{t}}\right)\left(2 k \bar{\phi}_{\bar{y}}-\frac{i k}{4 \hat{\omega}}\left(2-\kappa^{2}-i \bar{\eta}_{\bar{x}} \bar{\eta}_{\bar{x}}\right) \bar{\phi}_{\bar{y} \bar{t}}\right.}{\hat{\omega}-k \bar{\eta}_{\bar{x}} \bar{\phi}_{\bar{y}}}
\end{aligned}
$$

Only the secular terms linear in $\tilde{t}$ in $\tilde{\eta}^{(3)}$ are shown since only these terms contribute to the large-scale solution. Also, only the solution to $\tilde{\eta}^{(3)}$ is given here because we will calculate only $\tilde{\eta}$ directly; $\tilde{\phi}$ will then be determined in terms of $\tilde{\eta}$. The value of $F(\bar{x}, \bar{t})$, computed in Section 5 , has been used in calculating $g(\bar{x}, \bar{t})$.

\section{Equations for the large-scale solution}

Having found $\tilde{\eta}$, we may apply invariance conditions using the operators (2.9) to obtain equations for $A_{0}, A_{1}$, and $A_{2}$. The secular terms contributing to the large-scale solution are

$$
\begin{aligned}
\tilde{\eta}= & A_{0} E+\mu i\left(\tilde{x}-\omega_{k} \tilde{t}\right) A_{1} E-\frac{1}{2} \mu^{2}\left\{\left(\tilde{x}^{2}+\cdots\right)+i \omega_{k k} \tilde{t}\right\} A_{2} E \\
& +\sigma^{2} \tilde{t} A_{0} E\left(f+g\left|A_{0}\right|^{2}\right)+\cdots .
\end{aligned}
$$

Applying the $t$-invariance condition to (4.1), we find from the terms independent of the small-scale variables

$$
\sigma^{2} A_{0 \bar{t}}=-i \omega A_{0}-i \mu \omega_{k} A_{1}-\frac{1}{2} i \mu^{2} \omega_{k k} A_{2}+\sigma^{2} A_{0}\left(f+g\left|A_{0}\right|^{2}\right) .
$$


Applying the $x$-invariance condition, we find from setting terms proportional to $\tilde{x}$ and terms independent of $\tilde{x}$ and $\tilde{t}$ separately equal to zero that

$$
\begin{gathered}
\sigma^{2} A_{0 \bar{x}}=i k A_{0}+i \mu A_{1} \\
\sigma^{2} i k_{\bar{x}} A_{0}+i \mu \sigma^{2} A_{1 \bar{x}}+\mu k A_{1}+\mu^{2} A_{2}=0 .
\end{gathered}
$$

The first term in (4.4) is present because of the dependence of $k$ on $\bar{x}$ and $\bar{t}$. These equations may be simplified if we define

$$
B_{n}(\bar{x}, \bar{t}) e^{i \sigma^{-2} \theta(\bar{x}, \bar{t})}=A_{n}
$$

for $n=0,1,2$ and set

$$
\theta_{\bar{x}}=k, \quad \theta_{\bar{t}}=-\omega .
$$

Then (4.2), (4.3), and (4.4) become

$$
\begin{gathered}
\sigma^{2} B_{0 \bar{i}}=-i \mu \omega_{k} B_{1}-\frac{1}{2} i \mu^{2} \omega_{k k} B_{2}+\sigma^{2} B_{0}\left(f+g\left|B_{0}\right|^{2}\right) \\
\sigma^{2} B_{0 \bar{x}}=i \mu B_{1} \\
\sigma^{2} i \theta_{\bar{x} \bar{x}} B_{0}+i \mu \sigma^{2} B_{1 \bar{x}}+\mu^{2} B_{2}=0 .
\end{gathered}
$$

Eliminating $B_{1}$ and $B_{2}$ from these equations, we find a Ginzburg-Landau equation

$$
B_{0 \bar{t}}+\omega_{k} B_{0 \bar{x}}=\frac{1}{2} i \sigma^{2} \omega_{k k} B_{0 \bar{x} \bar{x}}+\left(f-\frac{1}{2} \omega_{k k} \theta_{\bar{x} \bar{x}}\right) B_{0}+g B_{0}\left|B_{0}\right|^{2} .
$$

To determine the dependence of $k$ and $\omega$ on $\bar{x}$ and $\bar{t}$, we use the dispersion relation (3.10) and the condition $\theta_{\bar{x} \bar{i}}=\theta_{\bar{i} \bar{x}}$, which is the wave conservation equation

$$
k_{\bar{t}}=-\omega_{\bar{x}} .
$$

Equation (4.11), together with (3.10), gives the rates of change of $k$ and $\omega$ at points moving with the speed $\omega_{k}$; since the dispersion relation involves the long-wave quantities $\bar{\eta}_{\bar{x}}$ and $\bar{\phi}_{\bar{x}}$, the values of $k$ and $\omega$ will change as the short-wave packet moves over the long wave. Alternatively, we may substitute (4.6) into the dispersion relation.

The solution for the surface elevation is then given by

$$
\bar{y}=\bar{\eta}(\bar{x}, \bar{t})+\sigma^{3} \bar{\eta}(\bar{x}, \bar{t}),
$$


where

$$
\tilde{\eta}=B_{0}(\bar{x}, \bar{t}) e^{i \sigma^{-2} \theta(\bar{x}, \bar{t})}+\text { c.c. }+\cdots
$$

with $B_{0}$ determined from (4.10) and $\theta$ from (4.6); $\bar{\eta}$ and $\bar{\phi}$ satisfy the long-wave equation given in the next section. Here we have followed the procedure described in Section 2, setting the variables $\tilde{x}$ and $\tilde{t}$ equal to zero and replacing the parameters $\bar{x}$ and $\bar{t}$ with variables denoted by the same symbols, scaled to the long-wave length and time scales. Thus, the solution is now described by a single quantity for each coordinate, rather than by the pairs of barred and tilded quantities. Since we have not defined separate variables for each length and time scale, the solutions $\tilde{\phi}$ and $\tilde{\eta}$ depend on the small parameters $\sigma$ and $\mu$ as well as on $\bar{x}$ and $\bar{t}$. While $\tilde{\phi}$ and $\tilde{\eta}$ have been scaled to be of order one, their derivatives with respect to $\bar{x}$ and $\bar{t}$ will contain terms that are large, due to the scales in the solution smaller than those of the long wave. The same is true of the solution $B_{0}$ : although the shortest length and time scales are accounted for through the exponential in (4.13), $B_{0}$ varies not only on the length and time scales of the long wave, but also on the somewhat shorter length scale of the wave-packet width.

We must now determine $\tilde{\phi}$. If we wanted $\tilde{\phi}$ in the entire flow region, it would be necessary to calculate its small-scale expansion and apply $x$ and $t$ invariance conditions. The problem is much simplified if we ask only for $\tilde{\phi}$ at the surface. The exact surface conditions, evaluated at $y=\eta$, are given by (2.3) and (2.4). Eliminating $\phi_{y}$ between the two equations, we find, in the original variables,

$$
\phi_{t}+\eta-\eta_{x x}\left(1+\eta_{x}^{2}\right)^{-3 / 2}+\frac{1}{2}\left(\phi_{x}^{2}+\eta_{t}^{2}+2 \eta_{t} \eta_{x} \phi_{x}+\eta_{x}^{2} \phi_{x}^{2}\right)=0
$$

at $y=\eta$. Dropping the subscript on $B_{0}$ for convenience,

$$
\tilde{\eta}=B e^{i \sigma^{-2} \theta}+\text { c.c. }+\cdots
$$

and we define $D$ by setting

$$
\tilde{\phi}=D e^{i \sigma^{-2} \theta}+\text { c.c. }+\cdots
$$

at $y=\eta$. Substituting in (2.5) and (2.6) and then in (4.14), equating terms that are $O(1)$ and proportional to $e^{i \sigma^{-2} \theta}$, and solving for $D$ results in

$$
D=i B \frac{\kappa^{3} \theta_{\bar{x}}^{2}+i \bar{\phi}_{\bar{y}}\left(\theta_{\bar{t}}+\bar{\phi}_{\bar{x}} \theta_{\bar{x}}\right)}{\theta_{\bar{t}}+\left(\bar{\phi}_{\bar{x}}+\bar{\eta}_{\bar{x}} \bar{\phi}_{\bar{y}}\right) \theta_{\bar{x}}}
$$


This defines $\tilde{\phi}$ at the surface in terms of the already determined $B$. The denominator is zero when the short wave has component of phase velocity tangential to the long-wave surface equal to the component of the long-wave surface velocity in the same direction. The disturbance ceases to exist as a wave when this is true.

The long-wave equation given in the following section also requires $\tilde{\phi}_{\bar{y}}$ at the surface, which is found by making the above substitutions in the kinematic boundary condition. The largest terms that are proportional to $e^{i \sigma^{-2} \theta}$ give

$$
\tilde{\phi}_{\bar{y}}=i \sigma^{-2}\left(\theta_{\bar{t}} B+\bar{\phi}_{\bar{x}} \theta_{\bar{x}} B+\eta_{\bar{x}} \theta_{\bar{x}} D\right) e^{i \sigma^{-2} \theta}+\text { c.c. }+\cdots .
$$

The expressions and the relation $\tilde{\phi}_{\bar{y} \bar{y}}=-\tilde{\phi}_{\bar{x} \bar{x}}$ allow all derivatives of $\tilde{\phi}$ to be determined at the surface.

Having specified $\tilde{\eta}$ and $\tilde{\phi}$ and their derivatives in terms of $B$, we turn to the initial and boundary conditions to be imposed. One could set up and solve a linear problem on the scale of the short waves describing the generation of the waves by a wavemaker or some other device. Here, however, we simply specify a distribution for $B$ at an initial time $\bar{t}=\bar{t}_{i}$, say $B\left(\bar{x}, \bar{t}_{i}\right)=B_{i}(\bar{x})$. We also require that the wave packet be localized, so that, at any time $\bar{t}, B(\bar{x}, \bar{t}) \rightarrow 0$ as $\bar{x} \rightarrow \pm \infty$. The length scale for $B_{i}$ is the length of the wave packet, which is $O(1)$ as a result of the choice $\mu=\sigma$ introduced following (3.13). Thus, the wave-packet length is taken to be of the same order as the wavelength of a capillary-gravity wave. The presence of a factor $\sigma^{2}$ in the amplitude equation (4.10) implies a distinguished limit for this case: since $B_{\bar{x}}=O\left(\sigma^{-1}\right)$, but $\theta_{\bar{x}}=O(1)$, all the terms on the right-hand side of (4.10) are of the same order. If instead we were to choose $\sigma^{2} \ll \mu \ll \sigma$ (longer wave packet), dispersion would be the dominant effect, whereas if $\sigma \ll \mu \ll 1$ (shorter wave packet), the dispersion term would be of higher order than the nonlinear and damping terms. In any case, the solution would be valid on the length and time scales of the long wave, due to our choices for $\lambda$ and $\tau$ in (2.10).

\section{Long-wave equation}

As discussed in Section 2, the terms in (2.3) and (2.4) comprising the long-wave equation are those containing no power of $e^{i \sigma^{-2} \theta}$. Thus, to the order of interest here, the terms contributing to the long-wave equation will be all those terms with only long-wave functions, and those terms quadratic in the short-wave functions. Note that each derivative of $\tilde{\eta}$ and $\tilde{\phi}$ is made larger by a factor $O\left(\sigma^{-2}\right)$, and includes higher-order terms. For example,

$$
\tilde{\phi}_{\bar{x} \bar{x}}=\sigma^{-4}\left\{-\theta_{\bar{x}}^{2} D+i \sigma^{2}\left(2 \theta_{\bar{x}} D_{\bar{x}}+\theta_{\bar{x} \bar{x}} D\right)+\cdots\right\} e^{i \sigma^{-2} \theta}+\text { c.c. }
$$

In the following, only terms $O\left(\sigma^{2}\right)$ relative to the first will be kept in these derivatives. 
The long-wave surface conditions (those in terms in the complete surface conditions independent of the fast exponential) are then

$$
\begin{gathered}
\bar{\eta}_{\bar{t}}-\bar{\phi}_{\bar{y}}+\bar{\eta}_{\bar{x}} \bar{\phi}_{\bar{x}}=2 \sigma^{2} k \hat{\omega} \frac{2 k \bar{\phi}_{\bar{y}}-\hat{\omega} \bar{\eta}_{\bar{x}}}{\hat{\omega}-k \bar{\eta}_{\bar{x}} \bar{\phi}_{\bar{y}}}|B|^{2}+o\left(\sigma^{2}\right) \\
\bar{\phi}_{\bar{t}}+\bar{\eta}-\sigma^{2} \bar{\eta}_{\bar{x} \bar{x}}\left(1+\bar{\eta}_{\bar{x}}^{2}\right)^{-3 / 2}+\frac{1}{2}\left(\bar{\phi}_{\bar{x}}^{2}+\bar{\phi}_{\bar{y}}^{2}\right)=-\sigma^{2} \frac{\hat{\omega}}{\left(\hat{\omega}-k \bar{\eta}_{\bar{x}} \bar{\phi}_{\bar{y}}\right)^{2}} \\
\times\left[\bar{\eta}_{\bar{x}}^{2} \hat{\omega}^{3}+2 \hat{\omega}^{2} k \bar{\eta}_{\bar{x}} \bar{\phi}_{\bar{y}}-\hat{\omega} k^{2} \bar{\phi}_{\bar{y}}^{2}+2 k^{3} \bar{\phi}_{\bar{y}}^{3}\right]|B|^{2}+o\left(\sigma^{2}\right)
\end{gathered}
$$

evaluated at $y=\bar{\eta}$. The terms retained on the right-hand side of these equations arise from products of short-scale quantities; the slowly varying terms subtracted from (3.3) and (3.4) (indicated by \langle\rangle ) are the first terms in short-scale expansions of the terms shown in (5.2) and (5.3).

The surface conditions (5.2) and (5.3), in conjunction with Laplace's equation for $\bar{\phi}$, could then be solved together with those of the preceding section to determine the evolution of the combined system of long and short waves. The region of validity of the combined system is limited by that of the short-wave equations, so the equations are valid on the length and time scales of the long wave. Since the terms in (5.2) and (5.3) giving the effect of the short wave on the long wave are small, it is not clear whether the short waves can have a significant effect on the long waves without going to larger length and time scales than those of the basic long wave. To go to these longer lengths and times, one would need to calculate more terms in the small-scale expansion of the short wave to increase its region of validity. (Of course, one could also make the long-wave amplitude smaller or the shortwave amplitude larger to increase the strength of the interaction.) Nevertheless, the combined equations could be solved with appropriate initial and boundary conditions for $\bar{\phi}$ and $\bar{\eta}$, and one could study the small effect of the short wave on the long wave. The equations for the complex amplitude of the short wave do not involve the smallest scale, so the numerical computations would not have to resolve the solution down to the scale of the short wave. These equations are thus, in effect a "model" for the short wave, analogous to the models used in turbulence computations.

It must not be supposed that the $O\left(\sigma^{2}\right)$ variations in $\bar{\phi}$ and $\bar{\eta}$ may be ignored. Variations of order $\sigma^{2}$ in $\bar{\phi}$ and $\bar{\eta}$ lead to $O\left(\sigma^{2}\right)$ changes in $\theta$, which affect the short wave significantly (cf. (4.5)). Thus, it would not be consistent to solve the combined system of equations while neglecting the right-hand side of (5.2) and (5.3). It is possible to solve the system sequentially, since (4.10) requires that $\theta, \bar{\phi}$, and $\bar{\eta}$ be known only to first order; one then may use the right-hand sides of (5.2) and (5.3) to get an $O\left(\sigma^{2}\right)$ correction to $\bar{\phi}$ and $\bar{\eta}$ and the dispersion relation to give an $O\left(\sigma^{2}\right)$ correction to $\theta$. 
While this set of equations has been derived for the case of infinite depth, it is in fact valid for large, but finite, depth as well, if appropriate boundary conditions are imposed on the long-wave potential. The infinite-depth solution for the short waves remains valid as long as the depth is large compared to the wavelength of the short wave.

\section{Discussion}

The result of our analysis is the system of equations involving the amplitude equation (4.10) coupled to the long-wave problem with surface conditions (5.2) and (5.3) solved in conjunction with Laplace's equation for $\bar{\phi}$ :

$$
\begin{aligned}
& B_{0 i}+\omega_{k} B_{0 \bar{x}}=\frac{1}{2} i \sigma^{2} \omega_{k k} B_{0 \bar{x} \bar{x}}+\left(f-\frac{1}{2} \omega_{k k} \theta_{\bar{x} \bar{x}}\right) B_{0}+g B_{0}\left|B_{0}\right|^{2} \\
& \bar{\eta}_{\bar{t}}-\bar{\phi}_{\bar{y}}+\bar{\eta}_{\bar{x}} \bar{\phi}_{\bar{x}}=2 \sigma^{2} k \hat{\omega} \frac{2 k \bar{\phi}_{\bar{y}}-\hat{\omega} \bar{\eta}_{\bar{x}}}{\hat{\omega}-k \bar{\eta}_{\bar{x}} \bar{\phi}_{\bar{y}}}|B|^{2}+o\left(\sigma^{2}\right) \\
& \bar{\phi}_{\bar{t}}+\bar{\eta}-\sigma^{2} \bar{\eta}_{\bar{x} \bar{x}}\left(1+\bar{\eta}_{\bar{x}}^{2}\right)^{-3 / 2}+\frac{1}{2}\left(\bar{\phi}_{\bar{x}}^{2}+\bar{\phi}_{\bar{y}}^{2}\right)=-\sigma^{2} \frac{\hat{\omega}}{\left(\hat{\omega}-k \bar{\eta}_{\bar{x}} \bar{\phi}_{\bar{y}}\right)^{2}} \\
& \times\left[\bar{\eta}_{\bar{x}}^{2} \hat{\omega}^{3}+2 \hat{\omega}^{2} k \bar{\eta}_{\bar{x}} \bar{\phi}_{\bar{y}}-\hat{\omega} k^{2} \bar{\phi}_{\bar{y}}^{2}+2 k^{3} \bar{\phi}_{\bar{y}}^{3}\right]|B|^{2}+o\left(\sigma^{2}\right)
\end{aligned}
$$

where the surface conditions are evaluated at $y=\bar{\eta}$. At the initial time, the long-wave surface elevation and velocity potential must be specified, along with the short-wave complex amplitude and wavenumber distribution. The long-wave surface elevation and velocity potential must satisfy whatever boundary conditions are appropriate for the particular flow being studied and the short-wave amplitude must decay to zero for sufficiently large distances from the wave packet.

We next consider the relationship between the present results and those of other authors.

(1) As stated earlier, the limit process chosen here for the long and short waves is the same as that considered by Benney [8]; consequently, the present results reduce to his if the long wave is linearized. The linearization is accomplished if $\bar{\eta}$ and $\bar{\phi}$ are reduced by a factor $\sigma^{4}$ and $B$ is made smaller by a factor $\sigma$. Benney's [8] equation for the amplitude of the short-wave potential (our $D$ ) has been expanded about $y=0$, and so we must use (4.18) to give the first two terms in the expansion, expand (4.17) in $\sigma^{2}$, and substitute the result in (4.10) to give an equation for $D$. The functions $f$ and $g$ are expanded in $\sigma^{2}$ as well, and $f$ is augmented by two terms $-i \sigma^{2} k \bar{\phi}_{\bar{x}}+i \sigma^{2} k^{-5 / 2} / 8$. The first of these replaces the second term on the right-hand side of (3.8), which is now small and can be discarded; the 
second is the second term in an expansion of the combined gravity-capillary dispersion relation under the present length and time scales. This second term would ordinarily appear in the next higher-order term in the perturbation series, but reducing the long-wave surface elevation and velocity potential has made the terms we have calculated as small as this previously neglected term, and so it must now be included for a consistent approximation. For the same reason, we include another term in a similar expansion of the group velocity in (4.10). Keeping terms up to $O\left(\sigma^{2}\right)$ in the coefficients yields the equation for $D$ :

$$
\begin{aligned}
D_{\bar{t}}+( & \left.\frac{3}{2} k^{1 / 2}-\frac{1}{4} \sigma^{2} k^{-3 / 2}\right) D_{\bar{x}}-\frac{3}{8} i \sigma^{2} k^{-1 / 2} D_{\bar{x} \bar{x}} \\
= & \left\{-\frac{i}{2} k^{-1 / 2}+\sigma^{2}\left(\frac{i}{8} k^{-5 / 2}+\frac{3}{2} k^{3 / 2} \bar{\phi}_{\bar{x} \bar{i}}-k \bar{\phi}_{\bar{y}}-i k \bar{\phi}_{\bar{x}}\right)\right\} D \\
& +\frac{i}{4} \sigma^{2} k^{5 / 2} D^{2} D^{*}-o\left(\sigma^{2}\right) .
\end{aligned}
$$

This equation is equivalent to that of Benney [8], in two dimensions. The linearization of the long wave has made the right-hand side of (6.1) smaller than that of (4.10); consequently, the region of validity is now larger, and $\bar{x}$ and $\bar{t}$ could be rescaled accordingly.

(2) Davey and Stewartson [7] treated the case of a single weakly nonlinear gravity-wave packet; the analogous result for a two-dimensional packet of capillary waves would be recovered by setting $\bar{\phi}=\bar{\eta}=0$ in (6.1). The present results also differ from those of Longuet-Higgins and Stewart [3] because their long and short waves are both gravity waves. The later numerical calculations of Longuet-Higgins [10] allow a finite-amplitude long wave; the formulation of Henyey et al. [11] includes short gravity-capillary waves as well. In each of these studies, the short wave is linear.

(3) It was mentioned above that the present may be viewed as a nonlinear extension of the work of Phillips [9]. Here we have included short-wave nonlinear effects, removed the restriction to a short-wave envelope fixed on the long wave, and extended the region of validity of the solution to the length and time scales of the long wave. (As shown explicitly in $[13,14]$, Phillips' results are valid up to length and time scales intermediate between those of the present short and long waves.)

(4) Zhang [13] and Zhang and Melville [14] have considered a problem similar to that of the present work, but both the long and short waves are gravity waves. Consequently, their limit process is different and their longwave slope must be made small in order that the long-wave convective effects and gravity effects may balance in the short-wave dispersion relation. (If the long-wave slope were of order one, the convective effects would dominate, the short-wave problem would be nondispersive to lowest order, and the resulting amplitude equation would presumably be something like 
the Korteweg-deVries equation.) The amplitude equation of Zhang and Melville thus contains effects of the long wave on the short-wave group velocity to first order, as in the present work, but the nonlinear term is unmodified by the long wave.

(5) Naciri and Mei [15] consider the interaction of a packet of smallamplitude, short, gravity waves with a finite-amplitude long, rotational, Gerstner wave. They use Lagrangian coordinates to deal with the expansion of the short-wave surface conditions about the long-wave surface, derive a Ginzburg-Landau equation for the complex amplitude of the short waves and use this equation to search for chaotic behavior. Since, as discussed in Section 2, it does not appear possible to set up a system of long and short gravity waves with finite-amplitude long waves and dispersive short waves, their formulation may not be fully self-consistent.

Some properties of the present equations may be inferred by looking at the special case of a short-wave wave packet fixed in a frame moving with the long wave. In this frame, the problem becomes a steady-state problem and (4.10) becomes

$$
\left(\omega_{k}-c\right) B_{\zeta}=\left(f-\frac{1}{2} \omega_{k k} \theta_{\zeta \zeta}\right) B+g B|B|^{2},
$$

where again we drop the subscript on $B_{0}$. The variables giving the position along the wave is $\zeta=\bar{x}-c \bar{t}$ ( $c$ is the long-wave phase speed) and we assume that the band-width of the short-wave wave packet is small enough that the $B_{\bar{x} \bar{x}}$ term may be neglected. In addition, the effect of the short waves on the long waves and the $O\left(\sigma^{2}\right)$ correction to the phase-function $\theta$ will not be considered. Equation (4.11), the wavenumber conservation relation, reduces to

$$
\omega_{\zeta}-c k_{\zeta}=0
$$

The integral of this equation, $\omega-c k=\omega_{0}$, is the relation given in [9]. Combined with the dispersion relation (3.10), this relation determines $\omega$ and $k$. Equation (6.2) may be integrated to give

$$
|B|=\exp \int_{\zeta_{0}}^{\zeta} h_{1 \mathrm{r}}(s) d s\left[\left|B_{0}\right|^{-2}-2 \int_{\zeta_{0}}^{\zeta} h_{2 \mathrm{r}}(s) \exp \left(2 \int_{\zeta_{0}}^{\zeta} h_{1 \mathrm{r}}\left(s^{\prime}\right) d s^{\prime}\right) d s\right]^{-1 / 2}
$$

and

$$
\text { ph } B=\text { ph } B_{0}+\int_{\zeta_{0}}^{\zeta}\left(h_{1 \mathrm{i}}(s)+h_{2 \mathrm{i}}(s)|B(s)|\right) d s,
$$


where $B_{0}$ is the value of $B$ at $\zeta=\zeta_{0}$. The functions $h_{1}$ and $h_{2}$ are given by

$$
\begin{aligned}
& h_{1}=\frac{f-\frac{1}{2} \omega_{k k} \theta_{\zeta \zeta}}{\omega_{k}-c} \\
& h_{2}=\frac{g}{\omega_{k}-c}
\end{aligned}
$$

and subscripts $r$ and $i$ denote real and imaginary parts, respectively.

The most striking feature of (6.4) is the possibility that the expression in brackets will be zero at some point and a square-root singularity in the amplitude will occur. We shall see in the numerical solutions to follow that this singularity does appear under certain conditions. Since the real part of $g$ is zero if there is no long wave, this singularity is entirely a result of the nonlinear interaction between the long and short waves.

Another singularity occurs if $\omega_{k}-c=0$ at some point $\zeta=\zeta_{\mathrm{b}}$. This is the capillary-blockage phenomenon described in [9]. The short capillary wave rides up the long gravity wave until the point $\zeta=\zeta_{\mathrm{b}}$, where the group velocity of the capillary wave is matched by the oppositely-directed surface velocity of the long wave and the short waves are prevented from traveling any further up the long wave. Near the blockage point $\zeta_{\mathrm{b}}$, the coefficient on the left-hand side of (6.2) is no longer slowly-varying and the multiple-scale analysis breaks down. This turning-point problem has been solved in the linear case by Shyu and Phillips [19]. The present solution may be used to characterize the singularity in the weakly nonlinear case (analogous to the solution of [9], but we do not attempt the nonlinear turning-point problem here.

Use of the dispersion relation and (6.3) give

$$
\omega_{k}-c \sim c_{1}\left(\zeta_{\mathrm{b}}-\zeta\right)^{1 / 2}
$$

for $\zeta_{\mathrm{b}}-\zeta$ small and positive. (We assume that the waves approach the blockage point from the left.) The constant $c_{1}$ is given by

$$
c_{1}=\left.\sqrt{2 \omega_{k k}}\left(\frac{3}{2} \kappa^{1 / 2} k^{3 / 2} k_{\zeta}+\bar{\phi}_{\zeta \zeta} k\right)^{1 / 2}\right|_{\zeta=\zeta_{\mathrm{b}}}
$$

Assuming that $f$ is finite at the blockage point, $h_{1}$ becomes, near the blockage point,

$$
h_{1} \sim \frac{1}{4} \frac{1}{\zeta_{\mathrm{b}}-\zeta}
$$

We note that $\theta_{\zeta \zeta}=k_{\zeta} \sim\left(\zeta_{\mathrm{b}}-\zeta\right)^{-1 / 2}$ contributed to this result. Use of the order-one part of (5.3) and the definition of the blockage point permits $h_{2 \mathrm{r}}$ 
to be simplified near the blockage point to

$$
h_{2 \mathrm{r}} \sim \frac{g_{\mathrm{br}}}{c_{1}}\left(\zeta_{\mathrm{b}}-\zeta\right)^{-1 / 2}
$$

where

$$
g_{\mathrm{br}}=-4\left[\kappa^{2} k^{2} \hat{\omega} \frac{\bar{\eta}_{\zeta}}{1+\frac{3}{2} \bar{\eta}_{\zeta}^{2}}\right]_{\zeta=\zeta_{\mathrm{b}}}
$$

Substituting these relations in (6.4) yields

$$
|B| \sim\left(-\frac{c_{1}}{2 g_{\mathrm{br}}}\right)^{1 / 2}\left(\zeta_{\mathrm{b}}-\zeta\right)^{-1 / 4}\left[-\ln \left(\zeta_{\mathrm{b}}-\zeta\right)\right]^{-1 / 2}
$$

The quantity in parenthesis in the first term on the right-hand side is positive if $c_{1}$ is positive (short waves are moving to right, toward $\zeta_{\mathrm{b}}$ ) and $g_{\text {br }}$ is negative ( $\hat{\omega}$ is taken to be positive and $\bar{\eta}_{\zeta}$ is positive if $\zeta_{\mathrm{b}}$ is to the left of the long-wave peak.) The effect of the weak nonlinearity is thus to weaken the singularity at the blockage point by a logarithmic factor. This solution also shows that, as far as the magnitude of the amplitude is concerned, all information about conditions to the left of $\zeta_{\mathrm{b}}$ is lost near the blockage point. Equation (6.12) is the weakly-nonlinear analog of Phillip's [9] linear characterization of the square-root singularity in energy at the blockage point.

Some numerical results are now presented to illustrate further properties of our equations. To simplify matters, the long waves will be assumed to be Stokes waves (linear waves with a nonlinear frequency shift) and the effect of the short waves on the long waves or the $O\left(\sigma^{2}\right)$ correction to the phase-function $\theta$ will not be considered. We again look only at especially narrow-banded wave packets. As above, the appropriate equations are (4.11) and (4.10) without the $B_{\vec{x} \bar{x}}$ term. The assumption that the short-wave envelope moves with the long waves is not made and the equations are solved numerically by means of the method of characteristics.

The initial wave-packet envelope is taken to be parabolic, with a maximum amplitude $\left|B_{0}\right|_{\max }$. The initial horizontal extent of the wave packet is from $\vec{x}=0.0$ to $\bar{x}=0.3 \pi$ and we take the initial wave number in the wave packet to be a constant, $k_{0}$. These initial conditions are arbitrary and are chosen merely to illustrate some qualitative characteristics of the solutions.

We shall look at the magnitude of the complex amplitude of the waves in the wave packet in a frame moving with the phase speed of the long wave and with the spatial variable scaled by a factor of $\pi:(\bar{x}-\alpha / \beta \bar{t}) / \pi$. The peak of the long wave is at $1 / 2$, the trough is at $3 / 2$ and the nodes are at 0 , 1 and 2. The magnitude will be scaled by the maximum initial amplitude: $|B| /\left|B_{0}\right|_{\max }$. The scaled amplitude of the long wave is $a$, its scaled wavenum- 
ber and frequency are $\alpha$ and $\beta$. Since the small parameter $\sigma$ has so far been defined only as a ratio of wavenumbers, it is permissible to set $\alpha=1$. The amplitude $a$ is then identical to the slope. In all the figures, the amplitude is plotted at $\bar{t}=0,1,2, \ldots$ and the wave packet is moving from left to right.

When the long-wave amplitude is very small and the short-wave amplitude is relatively small, the type of behavior first observed by LonguetHiggins and Stewart [11] is present: the short-wave amplitude increases at the peak of the long wave and decreases at the trough. In Figure 2 is plotted the results for $|B|_{\max }=0.2, k_{0}=0.9$, and $a \alpha=0.05$. We see that in addition to the increase and decrease in amplitude, the wave packet spreads due to dispersion of the waves in the packet.

For certain combinations of the parameters, this behavior can actually be reversed by the short-wave nonlinearity. In Figure 3 results are plotted for $\left|B_{0}\right|_{\max }=0.7, k_{0}=0.9$, and $a \alpha=0.05$. The short-wave amplitude decreases at the peak of the long wave and increases at the trough.

Capillary blockage is seen at larger long-wave amplitudes. In Figure 4, the parameters are $\left|B_{0}\right|_{\max }=0.4, k_{0}=0.7$, and $a \alpha=0.1$. Part of the packet is blocked, but the rest leaks through to the other side due to the variation in the blockage point for different members of the wave packet as a result of their differing wavenumbers.

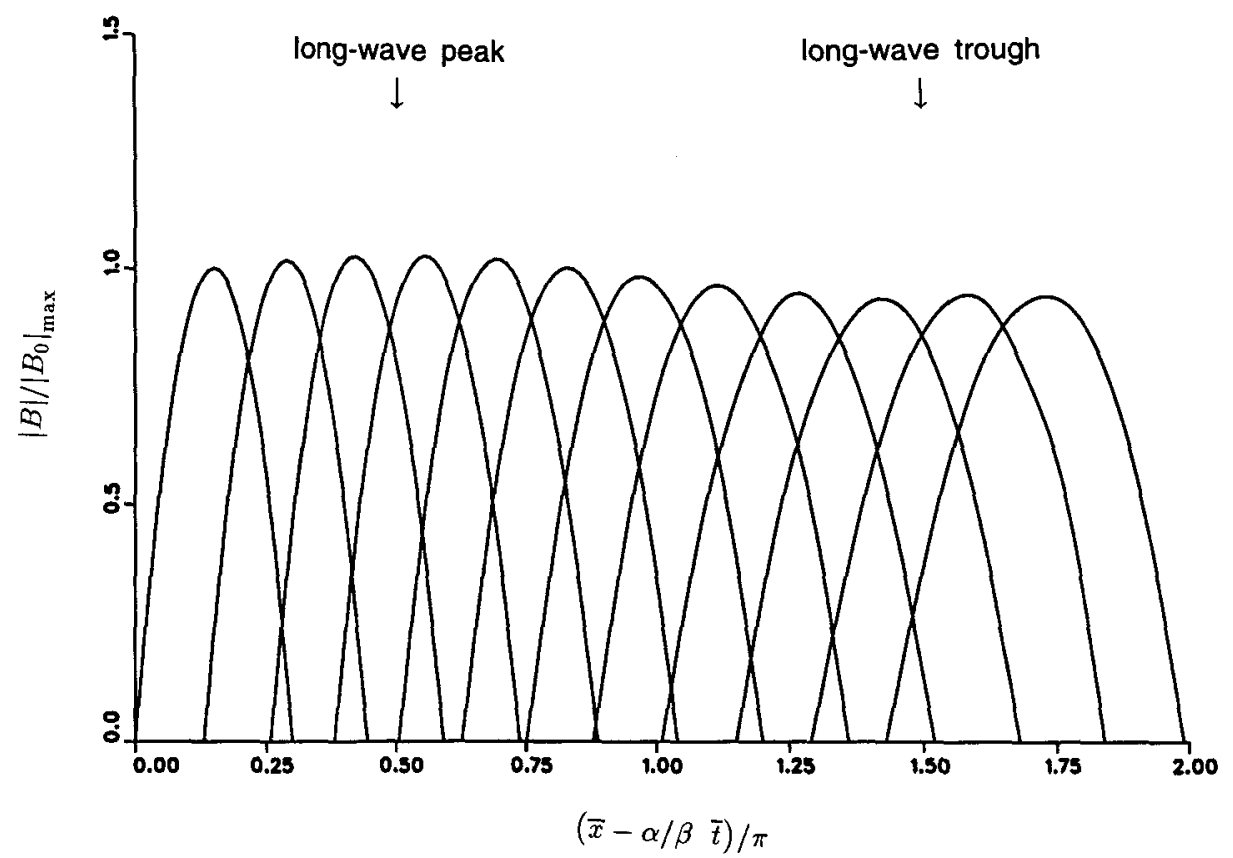

Figure 2. Magnitude of the short-wave amplitude for small-amplitude long wave and smallamplitude short wave, showing amplitude increase at peak, amplitude decrease at trough. Long-wave slope is 0.05 , short-wave initial conditions are $k_{0}=0.9,\left|B_{0}\right|_{\max }=0.2$. 


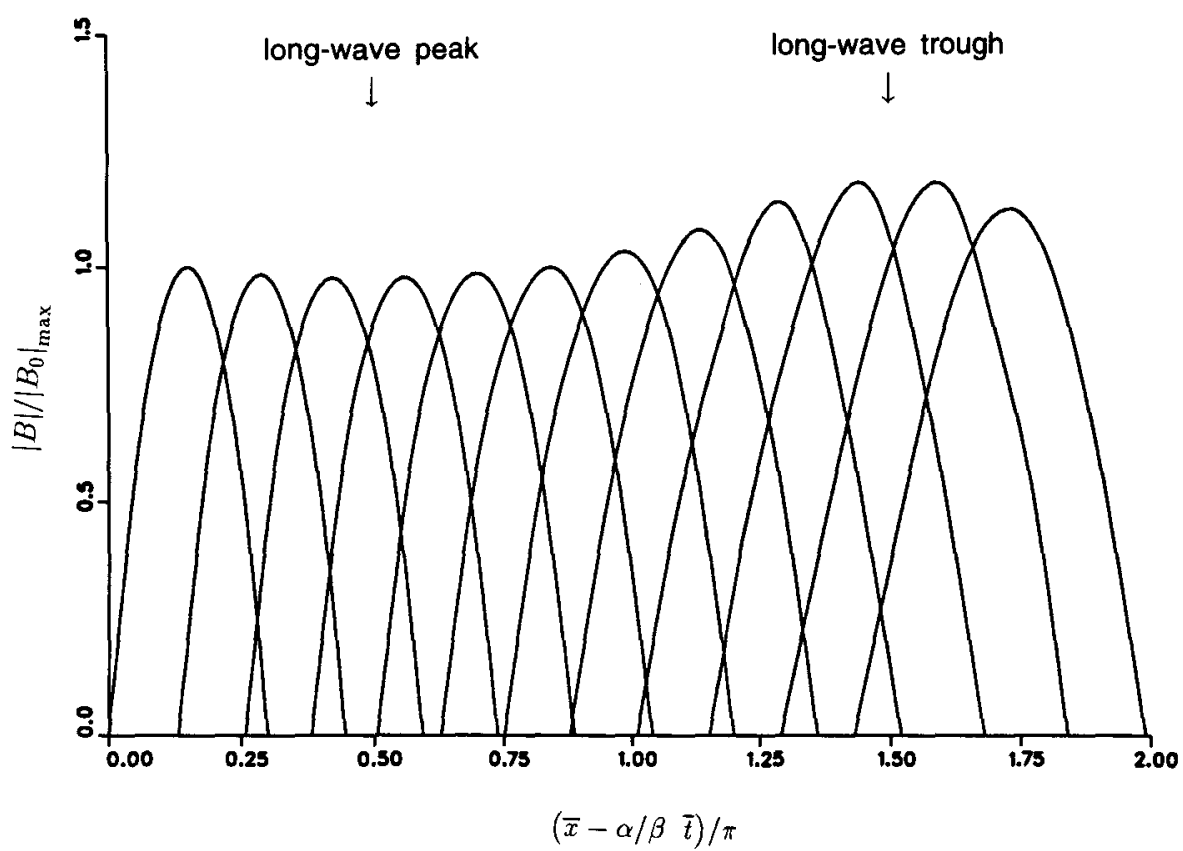

Figure 3. Short-wave amplitude when nonlinear effects become stronger, showing amplitude decrease at peak, increase at trough. Long-wave slope is 0.05 , short-wave initial conditions are $k_{0}=0.9,\left|B_{0}\right|_{\max }=0.7$.

Finally, if we set $\left|B_{0}\right|_{\max }=0.4, k_{0}=1.0$, and $a \alpha=0.3$, Figure 5 shows that the packet does not really become blocked, but spreads out until the leading edge reaches a point near the trough where the nonlinear singularity discussed above comes into play.

\section{Concluding remarks}

We have derived a system of equations describing the interaction of a packet of short, small-amplitude capillary waves with a long gravity wave with order-one slope. The system may be used to study this interaction, and, in particular, study the question raised in the introduction of how short waves reflect the properties of the long waves they travel over. The system could also be used to study short-wave instabilities of gravity waves, viewing the short waves as disturbance waves and determining whether they experience a net growth or decay as they pass over the gravity waves. These or similar equations might also permit the development of a scheme for numerical analysis where the effects of the short waves are included without actually resolving their small scales because the short waves may be characterized by $B$ and $\theta$, which depend only on the larger scales.

The invariance-condition method provided a systematic and straightforward approach to this problem; in particular, it made the computer evalua- 


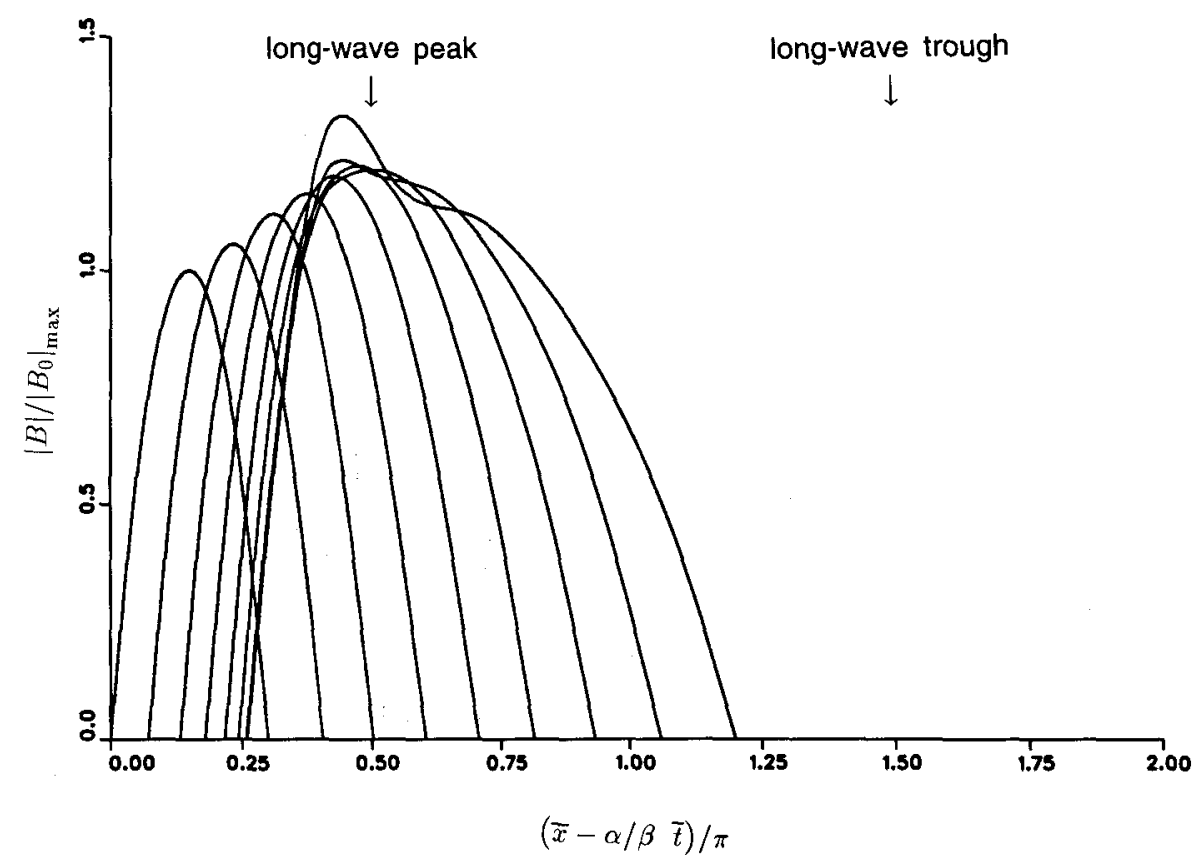

Figure 4. Short-wave amplitude when blockage occurs. Long-wave slope is 0.1 , short wave initial conditions are $k_{0}=0.7,\left|B_{0}\right|_{\max }=0.4$.

tion of the higher-order terms in the perturbation expansions especially convenient through the generating-function technique. It was also possible to solve the problem without adopting a special transformation of the coordinates, as was done by Phillips [9] and Zhang and Melville [19]. It is hoped that this approach will be of further use in problems such as this one involving the nonlinear propagation of waves in an active or inactive inhomogeneous medium with the inhomogeneities appearing at first order.

\section{Appendix}

The right-hand sides of (3.16) and (3.17) are

$$
\begin{gathered}
P^{(2)}=-2 \kappa^{2} \hat{\omega} k\left(i-\bar{\eta}_{\bar{x}}\right) A_{0}^{2} E^{2}+\text { c.c. } \\
Q^{(2)}=\kappa^{2} \hat{\omega}^{2}\left(1+4 i \bar{\eta}_{\bar{x}}\right) A_{0}^{2} E^{2}+\text { c.c. } \\
P^{(3)}=\left(\sum P_{m n}^{(3)} \tilde{t}^{m} \xi^{n}\right) A_{0} E+\frac{1}{2} i \kappa^{2} \hat{\omega} k^{2}\left(1+3 \kappa^{2}+i \kappa^{2} \bar{\eta}_{\bar{x}}\right) A_{0}^{2} A_{0}^{*} E+\text { c.c. } \\
Q^{(3)}=\left(\sum Q_{m n}^{(3)} \tilde{t}^{m \xi^{n}}\right) A_{0} E-\frac{1}{2} \kappa^{2} \hat{\omega} k\left(1+2 \kappa^{2}+i \kappa^{2} \bar{\eta}_{\bar{x}}\right) A_{0}^{2} A_{0}^{*} E+\text { c.c. }
\end{gathered}
$$




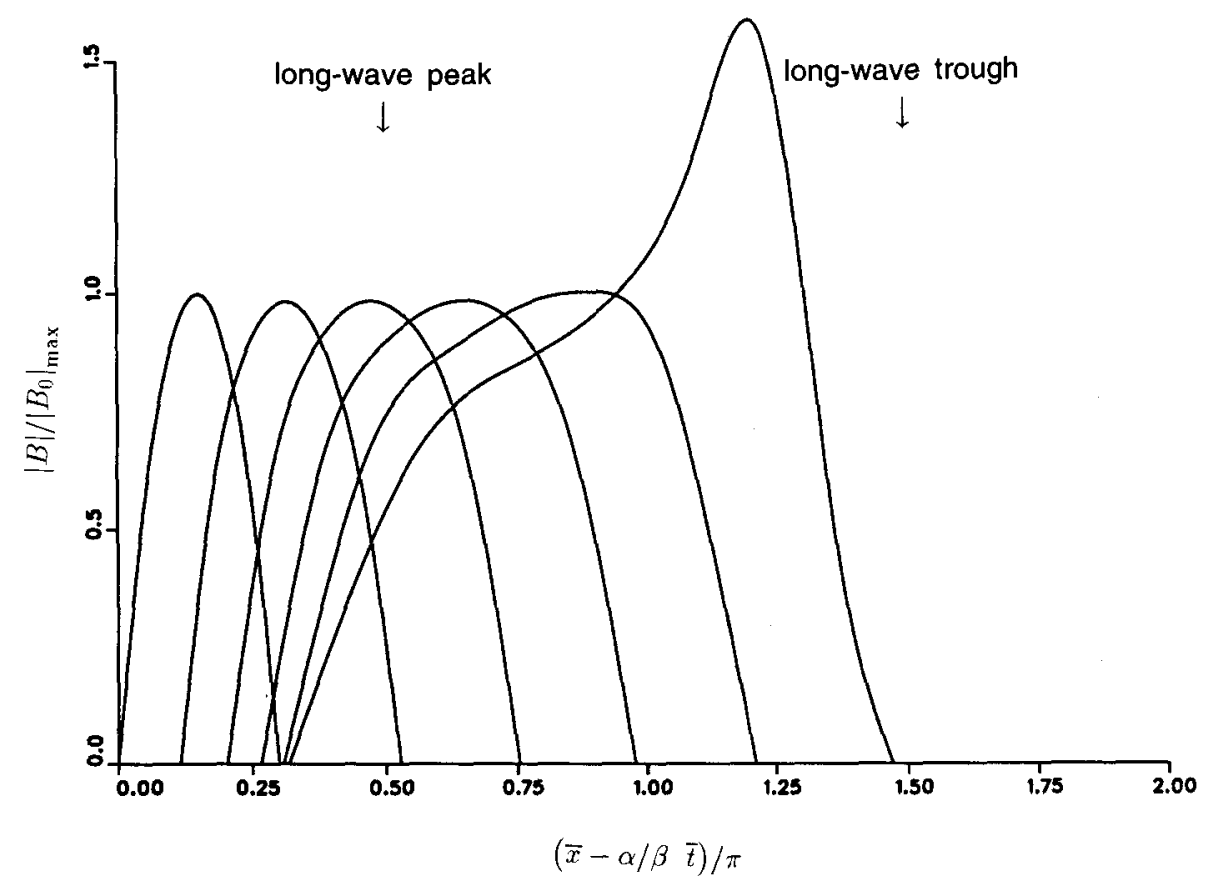

Figure 5. Short wave amplitude for case showing nonlinear singularity near trough. Long-wave slope is 0.3 , short-wave initial conditions are $k_{0}=1,\left|B_{0}\right|_{\max }=0.4$.

The summations are taken over both $m$ and $n$ such that $m, n \geq 0$ and $m+n \leq 2$. The quantities $P_{m n}^{(3)}$ and $Q_{m n}^{(3)}$ are

$$
\begin{aligned}
& P_{00}^{(3)}=-\bar{\phi}_{\bar{x} \bar{x}}-\bar{\eta}_{\bar{x}} \bar{\phi}_{\bar{x} \bar{y}}, \\
& P_{10}^{(3)}=-\frac{\hat{\omega}}{b}\left(\bar{\eta}_{\bar{x} \bar{t}}+\omega_{k} \bar{\eta}_{\bar{x} \bar{x}}\right)-i k\left(\bar{\eta}_{\bar{t}}+\omega_{k} \bar{\eta}_{\bar{x}}\right) \bar{\phi}_{\bar{x} \bar{y}}-i k\left(\bar{\phi}_{\bar{x} \bar{t}}+\omega_{k} \bar{\phi}_{\bar{x} \bar{x}}\right) \\
& P_{01}^{(3)}=-\frac{\hat{\omega}}{b} \bar{\eta}_{\bar{x} \bar{x}}-i k\left(\bar{\phi}_{\bar{x} \bar{x}}+\bar{\eta}_{\bar{x}} \bar{\phi}_{\bar{x} \bar{y}}\right), \quad P_{20}^{(3)}=-\frac{i \hat{\omega} k}{2 b}\left(\bar{\eta}_{i \bar{t}}+2 \omega_{k} \bar{\eta}_{\bar{x} \bar{x}}+\omega_{k}^{2} \bar{\eta}_{\bar{x} \bar{x}}\right) \\
& P_{11}^{(3)}=-\frac{i \hat{\omega} k}{b}\left(\bar{\eta}_{\bar{x} \bar{t}}+\omega_{k} \bar{\eta}_{\bar{x} \bar{x}}\right), \quad P_{02}^{(3)}=-\frac{i \hat{\omega} k}{2 b} \bar{\eta}_{\bar{x} \bar{x}} \\
& Q_{00}^{(3)}=-1-\bar{\phi}_{i \bar{y}}-\bar{\phi}_{\bar{x}} \bar{\phi}_{\bar{x} \bar{y}}-\bar{\phi}_{\bar{y}} \bar{\phi}_{\bar{y} \bar{y}}-3 i \kappa \kappa^{5} k \bar{\eta}_{\bar{x}} \bar{\eta}_{\bar{x} \bar{x}} \\
& Q_{10}^{(3)}=-\frac{\hat{\omega}}{b}\left[i\left(\bar{\eta}_{\bar{t}}+\omega_{k} \bar{\eta}_{\bar{x}}\right)\left(\bar{\phi}_{\bar{x} \bar{x}}-i \bar{\phi}_{\bar{x} \bar{y}}\right)+\bar{\phi}_{\bar{x} \bar{x}}-i \bar{\phi}_{\bar{y} \bar{t}}+\omega_{k} \bar{\phi}_{\bar{x} \bar{x}}-i \omega_{k} \bar{\phi}_{\bar{x} \bar{y}}\right]
\end{aligned}
$$




$$
\begin{gathered}
+3 \kappa^{5} k^{2} \bar{\eta}_{\bar{x}}\left(\bar{\eta}_{\bar{x} \bar{t}}+\omega_{k} \bar{\eta}_{\bar{x} \bar{x}}\right) \\
Q_{01}^{(3)}=-\frac{\hat{\omega}}{b}\left(1+i \bar{\eta}_{\bar{x}}\right)\left(\bar{\phi}_{\bar{x} \bar{x}}-i \bar{\phi}_{\bar{x} \bar{y}}\right)+3 \kappa^{5} k^{2} \bar{\eta}_{\bar{x}} \bar{\eta}_{\bar{x} \bar{x}} \\
Q_{20}^{(3)}=\frac{\hat{\omega}^{2}}{2 b}\left(\bar{\eta}_{\bar{i} \bar{t}}+2 \omega_{k} \bar{\eta}_{\bar{x} \bar{t}}+\omega_{k}^{2} \bar{\eta}_{\bar{x} \bar{x}}\right) \\
Q_{11}^{(3)}=\frac{\hat{\omega}^{2}}{b}\left(\bar{\eta}_{\bar{x} \bar{t}}+\omega_{k} \bar{\eta}_{\bar{x} \bar{x}}\right), \quad Q_{02}^{(3)}=\frac{\hat{\omega}^{2}}{2 b} \bar{\eta}_{\bar{x} \bar{x}}
\end{gathered}
$$

We denote the contribution of a term $\tilde{t}^{m} \xi^{n} E$ on the right-hand side of the kinematic surface condition to the secular part of $\tilde{\eta}$ linear in $\tilde{t}$ by $\gamma_{m n}$ and the contribution of such a term in the dynamic surface condition by $\delta_{m n}$. Then the results from the generating-function calculation are

$$
\begin{array}{lll}
\gamma_{00}=\frac{1}{2}, & \gamma_{10}=\frac{i}{4 \hat{\omega}}, & \gamma_{01}=0, \\
\gamma_{20}=\frac{1}{4 \hat{\omega}^{2}}, & \gamma_{11}=-\frac{3}{8 \hat{\omega} k}, & \gamma_{02}=\frac{3}{16 k^{2}}, \\
\delta_{00}=\frac{i k}{2 \hat{\omega}}, & \delta_{10}=\frac{k}{4 \hat{\omega}^{2}}, & \delta_{01}=-\frac{1}{4 \hat{\omega}}, \\
\delta_{20}=-\frac{i k}{4 \hat{\omega}^{3}}, & \delta_{11}=\frac{i}{2 \hat{\omega}^{2}}, & \delta_{02}=-\frac{9 i}{16 \hat{\omega} k}
\end{array}
$$

\section{Acknowledgments}

The authors thank J. T. Stuart for his helpful comments on an earlier version of this paper. This research was supported in part under the Program in Ship Hydrodynamics at The University of Michigan, funded by the University Research Initiative of the Office of Naval Research, Contract No. N000184-86-K-0684. S.L.W.'s work was also supported in part by the U.S. Army through the Mathematical Sciences Institute at Cornell University and in part by DARPA URI Grant N00014-86-K0754 to the Center for Fluid Mechanics, Turbulence, and Computation at Brown University.

\section{References}

1. G. G. STOKES, On the theory of oscillatory waves, Trans. Cambridge Philos. Soc. 8:441-455 (1847).

2. O. M. Phillips, Remote sensing of the sea surface, Ann. Rev. Fluid Mech. 20:89-109 (1988).

3. M. S. Longuet-Higgins AND R. W. STEWART, Changes in the form of short gravity waves on long waves and tidal currents, J. Fluid Mech. 8:565-583 (1960). 
4. V. E. ZaKHaRov, Stability of periodic waves of finite amplitude on the surface of deep fluid, J. Appl. Mech. Tech. Phys. 9:190-194 (1968).

5. D. J. BENNEY AND G. J. RoskEs, Wave instabilities, Stud. Appl. Math. 48:377-385 (1969).

6. D. J. BENNEY AND K. CHOw, An alternative approach to nonlinear instabilities in hydrodynamics, Stud. Appl. Math. 73:261-267 (1985).

7. A. DAVEY AND K. STEWARTSON, On three-dimensional packets of surface waves, Proc. Roy. Soc. (Lond.) A 338:101-110 (1974).

8. D. J. Benney, Significant interactions between small and large scale waves, Stud. Appl. Math. 55:93-106 (1976).

9. O. M. PHILLIPS, The dispersion of short wavelets in the presence of a long wave, J. Fluid Mech. 107:465-485 (1981).

10. M. S. LONGUET-HIGGINS, The propagation of short surface waves on longer gravity waves, J. Fluid Mech. 177:293-306 (1987).

11. F. S. Henyey, D. B. Creamer, K. B. Dysthe, R. L. Schult, and J. A. Wright, The energy and action of small waves riding on large waves, J. Fluid Mech. 189:443-462 (1988).

12. R. GRIMSHAw, The modulation of short gravity waves by long waves or currents, J. Austral. Math. Soc. Ser. B 29:410-429 (1988).

13. J. ZHANG, Nonlinear Interaction between Surface Water Waves, Ph.D. Thesis, Massachusetts Institute of Technology, 1987.

14. J. ZHANG AND W. K. MELVILLE, Evolution of weakly nonlinear short waves riding on long gravity waves, J. Fluid Mech. 214:321-346 (1990).

15. M. NACIRI AND C. C. MEI, Evolution of a short surface wave on a very long surface wave of finite amplitude, J. Fluid Mech. 235:415-452 (1992).

16. S. L. WoOdRufF, A Singular-Perturbation Analysis of Weakly Nonlinear Evolution of Long and Short Water Waves and Waves in Boundary Layers, Ph.D. Thesis, The University of Michigan, 1987.

17. S. L. WOODRUFF, The use of an invariance condition in the solution of multiple-scale problems, Stud. Appl. Math. 90:225-248 (1993).

18. J. Kevorkian AND J. D. Cole, Perturbation Methods in Applied Mathematics, Springer, New York, 1981.

19. J.-H. ShyU AND O. M. PHILUIPS, The blockage of gravity and capillary waves by longer waves and currents, J. Fluid Mech. 217:115-141 (1990).

\author{
BROWN UNIVERSITY \\ UNIVERSITY OF MICHIGAN
}

(Received June 10, 1993) 